\title{
Genome-wide Identification and Expression Analysis of the CDPK Gene Family in Grape, Vitis spp
}

Kai Zhang ${ }^{1,2 \dagger}$, Yong-Tao Han ${ }^{1,2 \dagger}$, Feng-Li Zhao ${ }^{1,2}$, Yang Hu ${ }^{1,2}$, Yu-Rong Gao ${ }^{1,2}$, Yan-Fei Ma ${ }^{1,2}$, Yi Zheng ${ }^{3}$, Yue-Jin Wang ${ }^{1,2}$ and Ying-Qiang Wen ${ }^{1,2^{*}}$

\begin{abstract}
Background: Calcium-dependent protein kinases (CDPKs) play vital roles in plant growth and development, biotic and abiotic stress responses, and hormone signaling. Little is known about the CDPK gene family in grapevine.

Results: In this study, we performed a genome-wide analysis of the $12 \mathrm{X}$ grape genome (Vitis vinifera) and identified nineteen CDPK genes. Comparison of the structures of grape CDPK genes allowed us to examine their functional conservation and differentiation. Segmentally duplicated grape CDPK genes showed high structural conservation and contributed to gene family expansion. Additional comparisons between grape and Arabidopsis thaliana demonstrated that several grape CDPK genes occured in the corresponding syntenic blocks of Arabidopsis, suggesting that these genes arose before the divergence of grapevine and Arabidopsis. Phylogenetic analysis divided the grape CDPK genes into four groups. Furthermore, we examined the expression of the corresponding nineteen homologous CDPK genes in the Chinese wild grape (Vitis pseudoreticulata) under various conditions, including biotic stress, abiotic stress, and hormone treatments. The expression profiles derived from reverse transcription and quantitative PCR suggested that a large number of VpCDPKs responded to various stimuli on the transcriptional level, indicating their versatile roles in the responses to biotic and abiotic stresses. Moreover, we examined the subcellular localization of VpCDPKs by transiently expressing six VpCDPK-GFP fusion proteins in Arabidopsis mesophyll protoplasts; this revealed high variability consistent with potential functional differences.
\end{abstract}

Conclusions: Taken as a whole, our data provide significant insights into the evolution and function of grape CDPKs and a framework for future investigation of grape CDPK genes.

Keywords: Grapevine (Vitis vinifera L.) (Vitis pseudoreticulata), CDPK, Synteny analysis, Phylogenetic tree, Subcellular localization, expression profiles

\section{Background}

Calcium $\left(\mathrm{Ca}^{2+}\right)$, a universal second messenger in eukaryotes, mediates stimulus-response coupling in the regulation of diverse cellular functions $[1,2]$. Various extracellular stimuli elicit specific calcium signatures that can be recognized by different calcium sensors. The three main classes

\footnotetext{
* Correspondence: wenyq@nwsuaf.edu.cn

${ }^{\dagger}$ Equal contributors

'State Key Laboratory of Crop Stress Biology for Arid Areas and College of Horticulture, Northwest A\&F University, Yangling 712100Shaanxi, People's Republic of China

${ }^{2}$ Key Laboratory of Horticultural Plant Biology and Germplasm Innovation in Northwest China, Ministry of Agriculture, Yangling 712100Shaanxi, People's Republic of China

Full list of author information is available at the end of the article
}

of $\mathrm{Ca}^{2+}$ sensors identified in plants are: CaMs (calmodulins) and CaM-like proteins, CBLs (calcineurin B-like proteins), and CDPKs (calcium-dependent protein kinases) [3-5]. The CDPKs, also termed CPKs, consist of a variable $\mathrm{N}$-terminal domain, a conserved serine/threonine kinase domain, an auto-inhibitory junction region, and a Cterminal regulatory CaM-like domain [6]. Unlike the other $\mathrm{Ca}^{2+}$ sensors, CDPKs have both $\mathrm{Ca}^{2+}$ sensing and responding activities due to their unique, CaM-like domain and protein kinase domain, which convert upstream $\mathrm{Ca}^{2+}$ signals into downstream phosphorylation events and cellular responses $[7,8]$.

\section{Biomed Central}

(c) 2015 Zhang et al. This is an Open Access article distributed under the terms of the Creative Commons Attribution License (http://creativecommons.org/licenses/by/4.0), which permits unrestricted use, distribution, and reproduction in any medium, provided the original work is properly credited. The Creative Commons Public Domain Dedication waiver (http:// creativecommons.org/publicdomain/zero/1.0/) applies to the data made available in this article, unless otherwise stated. 
Genome-wide analysis, together with comparative genomics, provides an effective way to understand the structures and functions of members of a gene family, using the insights gained from evolutionary relationships and experimental data. The original, systematic report on the CDPK gene family in Arabidopsis thaliana identified 34 $C D P K$ gene family members [9], and was followed by research in rice (Oryza sativa) [10] and wheat (Triticum aestivum) [11]. Recently, genome-wide analyses of the $C D P K$ gene family have been reported in maize (Zea mays L) [12] and poplar (Populus trichocarpa) [13]. Meanwhile, more and more investigations of $C D P K$ genes have also involved horticultural plants, such as alfalfa [14], potato [15], strawberry [16], and tomato [17].

Work in Arabidopsis showed that CDPKs function in immune and stress signaling, growth and development, and hormone responses. AtCPKs play vital parts in immune signaling pathways; for example, AtCPK1 activates NADPH oxidase, resulting in an oxidative burst [18], and phosphorylates PAL (Phenylalanine ammonia-lyase) resulting in accumulation of salicylic acid (SA) [19]. AtCPK4/5/6/11 phosphorylates a specific subgroup of WRKY transcription factors, WRKY8/28/48, which participate in NLR-dependent restriction of pathogen growth. In addition, AtCPK5 activates $\mathrm{RBOH}$ to induce a reactive oxygen species (ROS) burst [20,21]. CPKs also function in the response to abiotic stress. For example, AtCPKs help to enhance drought tolerance by responding to abscisic acid (ABA), leading to induction of expression of genes such as $A t C P K 4 / 11$ [22], and stomatal closure via induction of AtCPK3/6 [23]. AtCPK4/11 [22] and $A t C P K 23$ [24] trigger plant salt tolerance via controlling of $\mathrm{K}^{+}$channels. In hormone signaling, studies have systematically examined ABA [25, 26], and a few studies have examined MeJA [27], SA [19], and ethylene [28]. The rich sequence and functional information from Arabidopsis enables us to extrapolate the functions of the orthologous genes in other species.

Grapevine is one of the most important fruit crops in the world. However, most cultivated grapevine varieties (Vitis vinifera) are susceptible to many pathogens and are sensitive to abiotic stresses. By contrast, Chinese wild grapevine (Vitis pseudoreticulata) accession Baihe-35-1 has demonstrated resistance to multiple diseases and to various environmental stresses [29, 30]. Previous examination of the CDPK gene family in Vitis vinifera identified 17 members [31], and in Vitis amurensis isolated 13 members [32]. However, prior work focused on evolutionary relationships with only a few transcriptional analyses in some tissues and developmental stages. In this study, we employed bioinformatics and publicly available data to identify and analyse grape $C D P K$ genes on a genome-wide scale in Vitis pseudoreticulata, identifying two more members. Furthermore, we measured the expression of the $C D P K$ genes $(V p C D P K s)$ in Chinese wild grapevine Vitis pseudoreticulata accession Baihe-35-1 in response to various biotic and abiotic stresses as well as multiple phytohormone treatments. In addition, we showed that the VPCDPKs have different subcellular localizations when transiently expressed in Arabidopsis mesophyll protoplasts. More significantly, comparing Arabidopsis and grapevine CDPK gene structures, evolution, and experimental data provides insights on the functions of $V p C D P K s$. Our results provide a set of potential candidate $C D P K$ genes for future genetic modification of pathogen resistance and stress tolerance in grapevine.

\section{Results}

\section{Characteristics of grape CDPK genes}

We identified $C D P K$ genes by searching the Pfam database and obtained the HMM (Hidden Markov Model) profiles of protein kinase domain Pkinase (PF00069) and EF-hand domain EF-hand_7 (PF13499). Then we used BLAST-P to search the $12 \mathrm{X}$ grapevine $(V$. vinifera) genome using Pkinase and EF-hand HMM profiles. We also performed BLAST-P searches at NCBI using full-length amino acid of putative grape $C D P K$ genes. After that, we identified a bunch of candidates (data not shown). By removing incomplete gene sequences, transcripts of the same genes, and redundant sequences, we identified nineteen non-redundant $C D P K$ genes in the grape genome (Table 1). Among those with alternative splice variants, we selected the longest variant for further analysis. The sequences of the nineteen grape CDPKs were submitted to InterPro and SMART databases to confirm their Pkinase domains and EF-hand domains. Previous work identified seventeen CDPK genes in grapevine [31]. The seventeen genes were named based on their distributions and relative linear orders on the respective chromosomes. On this basis, we added two members (GSVIVT01025745001 and GSVIVT01027353001) and named them $V v C D P K 18$ and $V \nu C D P K 19$ according to their chromosomal locations. The nineteen putative $C D P K$ genes mapped on eleven grape chromosomes (Fig. 1). Among them, eight chromosomes possess one CDPK gene, three possess two CDPK genes, and Chr. 8 possesses five $C D P K$ genes.

Characteristics of the nineteen $C D P K$ genes are shown in Table 1. The lengths of the gene sequences vary widely, from 3.103 to $21.654 \mathrm{~kb}$, but the numbers of predicted amino acids are relatively similar for most genes, around 520 to 580, except VvCDPK16 with 626 amino acids. Fifteen of the nineteen CDPKs are predicted to be palmitoylated and eight of the nineteen CDPKs are predicted to be myristoylated. The predicted proteins for six CDPK genes have both $\mathrm{N}$-terminal myristoylation sites and palmitoylation sites. Nine CDPK genes encode proteins with only palmitoylation sites and two $(V v C D P K 3$ and $V \nu C D P K 7)$ have only myristoylation sites. Besides 
Table 1 Characteristics of Grape CDPK genes

\begin{tabular}{|c|c|c|c|c|c|c|c|c|c|c|c|}
\hline Name & Locus ID ${ }^{a}$ & Length (kb) & No. of aa & N-Term. $a a^{b}$ & N-Myr. ${ }^{\mathrm{C}}$ & N-Pal. ${ }^{d}$ & No. of $\mathrm{EFs}^{\mathrm{e}}$ & Chr. & Group & Homologs in $V p .^{f}$ & Identity $^{9}(\%)$ \\
\hline VVCDPK1 & GSVIVT01019446001 & 9.175 & 528 & MGNCNGLP & $\mathrm{N}$ & Y & 4 & 2 & $\|$ & VpCDPK1 & - \\
\hline VVCDPK2 & GSVIVT01023866001 & 5.366 & 561 & MGNTCRGS & $\mathrm{N}$ & Y & 4 & 3 & I & VpCDPK2 & 99.5 \\
\hline VVCDPK3 & GSVIVT01018778001 & 6.278 & 558 & MGACLSAT & Y & N & 4 & 4 & IV & VpCDPK3 & 99.5 \\
\hline$V_{V} C D P K 4$ & GSVIVT01010743001 & 16.91 & 554 & MGGCISMP & Y & Y & 4 & 5 & III & VpCDPK4 & - \\
\hline VVCDPK5 & GSVIVT01025249001 & 6.444 & 518 & MGNCCASP & $\mathrm{N}$ & Y & 4 & 6 & III & VpCDPK5 & 97.7 \\
\hline VVCDPK6 & GSVIVT01037295001 & 3.103 & 534 & MGNCCSQG & Y & Y & 4 & 6 & $\|$ & VpCDPK6 & - \\
\hline$V_{V} C D P K 7$ & GSVIVT01000238001 & 7.807 & 540 & MGMYTSKD & Y & N & 4 & 7 & I & VpCDPK7 & - \\
\hline VVCDPK8 & GSVIVT01022524001 & 11.179 & 568 & MGNTCVGGP & $\mathrm{N}$ & Y & 4 & 8 & । & VpCDPK8 & - \\
\hline VVCDPK9 & GSVIVT01022606001 & 3.947 & 580 & MGNNCVGS & $\mathrm{N}$ & Y & 4 & 8 & । & VpCDPK9 & 99.3 \\
\hline VVCDPK10 & GSVIVT01011167001 & 18.233 & 527 & MGNCCRSP & $\mathrm{N}$ & Y & 4 & 8 & III & VpCDPK10 & 99.8 \\
\hline VVCDPK11 & GSVIVT01033306001 & 5.504 & 526 & MGNCCVTP & $\mathrm{N}$ & Y & 4 & 8 & III & VpCDPK11 & 100 \\
\hline VVCDPK12 & GSVIVT01012730001 & 8.352 & 545 & MGCFSSKE & Y & Y & 4 & 10 & $\|$ & VpCDPK12 & - \\
\hline VVCDPK13 & GSVIVT01001931001 & 6.935 & 569 & MGNTCEGGP & $\mathrm{N}$ & Y & 4 & 13 & । & VpCDPK13 & - \\
\hline VVCDPK14 & GSVIVT01008077001 & 9.065 & 552 & MGNCIACV & Y & Y & 4 & 17 & III & VpCDPK14 & - \\
\hline VVCDPK15 & GSVIVT01008749001 & 5.975 & 523 & MGFCFSRP & Y & Y & 4 & 18 & $\|$ & VpCDPK15 & - \\
\hline VVCDPK16 & GSVIVT01034489001 & 21.654 & 626 & MVIKTKIS & N & N & 4 & 18 & 1 & VpCDPK16 & - \\
\hline VVCDPK17 & GSVIVT01037652001 & 5.084 & 536 & MGICLSKG & Y & Y & 4 & 19 & $\|$ & VpCDPK17 & - \\
\hline VVCDPK18 & GSVIVT01025745001 & 8.344 & 575 & MGLCQGKP & ND & Y & 2 & 8 & IV & VpCDPK18 & - \\
\hline VVCDPK19 & GSVIVT01027353001 & 3.399 & 520 & MGQETRRL & $\mathrm{N}$ & $\mathrm{N}$ & 3 & 13 & IV & VpCDPK19 & 99.8 \\
\hline
\end{tabular}

a IDs are available in the Grape Genome Browser (12X) (http://www.genoscope.cns.fr/externe/GenomeBrowser/Vitis/)

${ }^{b}$ First eight amino acids at the $\mathrm{N}$-terminal of the corresponding protein. The amino acids underlined indicate putative palmitoylation sites

c The myristoylation sites were predicted by the Myristoylator program (http://web.expasy.org/myristoylator/). ND, not determined

d The palmitoylation sites were predicted by CSS-Palm 3.0 (http://csspalm.biocuckoo.org/)

e Number of EF-hands were predicted by InterPro (http://www.ebi.ac.uk/interpro/scan.html)

${ }^{f}$ Homologous CDPK genes in Chinese wild grapevine Vitis pseudoreticulata

${ }^{g}$ Sequence identity calculated with amino acid sequences using Vector NTI

these, $V v C D P K 16$ and $V \nu C D P K 19$ have neither myristoylation nor palmitoylation sites. Typical CDPKs have four EF-hands, but not necessarily $[9,33]$. The seventeen previously identified CDPKs were predicted to each have four EF-hands. In addition, the two new members, VvCDPK18 and VvCDPK19 were predicted to have two EF-hands and three EF-hands, respectively.

\section{Grape CDPK genes possess typical domains but complicated intron-exon organization}

The predicted protein sequences of the nineteen grape CDPKs contain the four typical CDPK domains, including the $\mathrm{N}$-terminal variable domain, the protein kinase domain, the junction domain, and the calmodulin-like domain (data not shown). The intron-exon organization can indicate the evolutionary relationships within multi-gene families [34]. As shown in Fig. 2, grape CDPK genes can be divided into four groups (I-IV) on the basis of the Arabidopsis classification [9]. Most of the grape CDPK genes have seven or eight exons, six or seven introns, and clear intron phase patterns. Group I members have seven exons, except $V v C D P K 16$ with ten exons, and their intron phases, with respect to the open reading frame, occur in the same pattern, with type- 2 intron phases at the first three positions from the beginning and type- 0 intron phases at the last three positions. All of the Group II members contain eight exons and also share the same intron phase pattern. Compared with the Group I members, Group II members have an additional exon. Group III can be divided into two subgroups. Subgroup 1 shares the same intron-exon organization as most of the Group I members, such as $V v C D P K 10$ and $V v C D P K 14$, but subgroup 2 members, such as $V \nu C D P K 4, V \nu C D P K 5$, and $V v C D P K 11$, have one more exon at the first position and one more type- 0 phase intron. Compared with the other three groups, Group IV has a complicated intron-exon organization with differing numbers of exons and intron phases. Clustering the intron-exon structures of the nineteen $C D P K$ genes by an unrooted phylogenetic tree suggests a connection between intron-exon structures and evolutionary relationships.

\section{Gene duplication and synteny analysis of grape CDPK genes}

Gene duplication and divergence are important in gene family expansion [35] and in the evolution of novel 

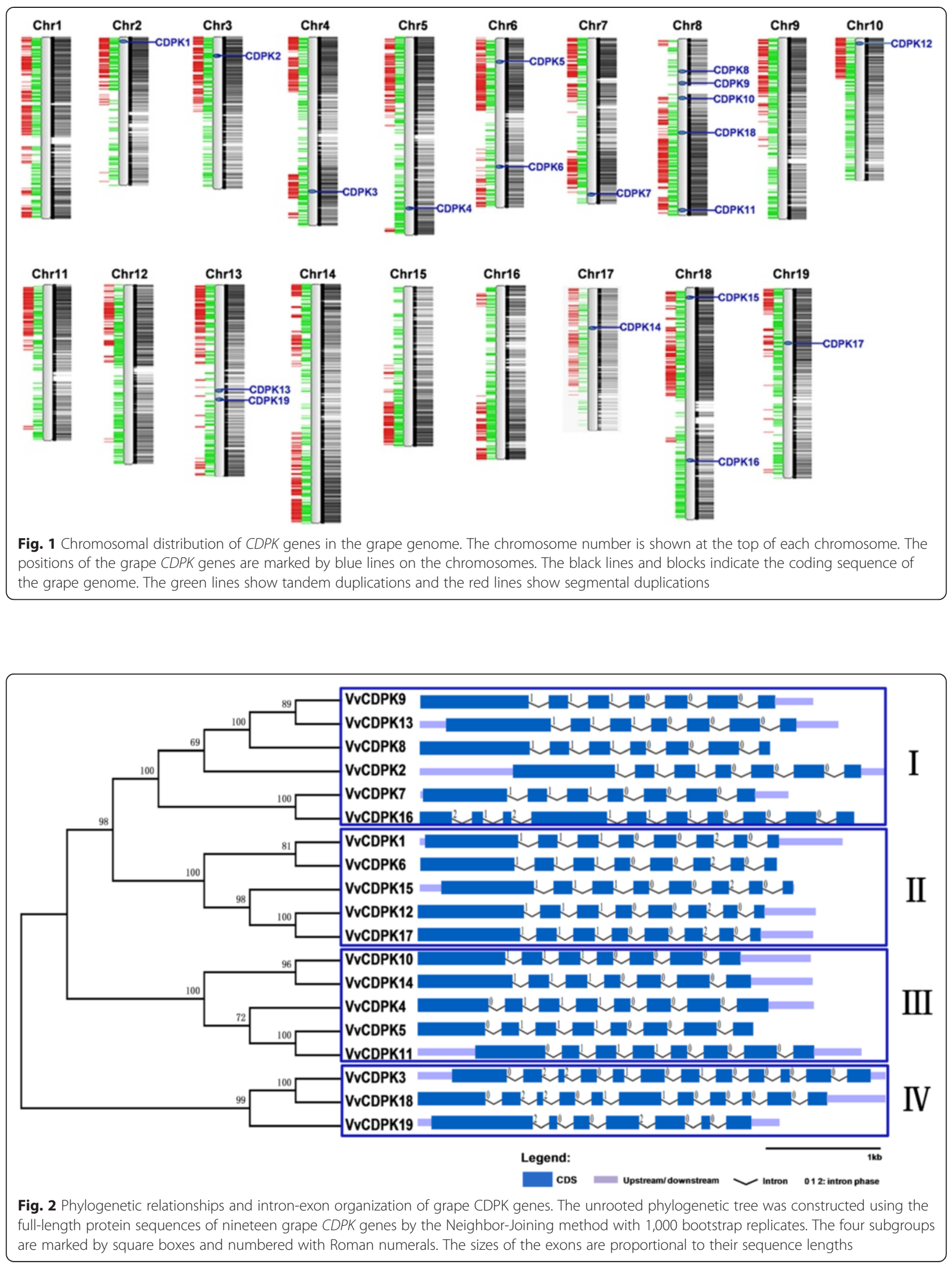
functions [36]. Grapevine has undergone whole-genome duplications during its evolutionary history [37]. To examine the effect of duplications on the CDPK gene family, we obtained tandem duplication and segmental duplication gene pairs from PGDD (Plant Genome Duplication Database) and visualized them using Circos. In this study, we identified two segmental duplication pairs of grape $C D P K$ genes $(V v C D P K 5$ and $V \nu C D P K 11, V \nu C D P K 12$ and $V v C D P K 17$ ) (Fig. 3) but did not find tandem duplication events among the grape $C D P K$ genes.

To further explore the origin and evolution of grape $C D P K$ genes, we investigated the syntenic relationship between grapevine and Arabidopsis. The synteny analysis showed that grape CDPK genes can be divided into two types (Fig. 3, Additional file 1. The first type of syntenic genes has a single grape gene that corresponds to a single Arabidopsis gene, including VvCDPK2-AT4G35310 (AtCP K5), VvCDPK4-AT2G31500 (AtCPK24), VvCDPK5-AT5G 12480 (AtCPK7), VvCDPK10-AT3G51850 (AtCPK13), $V \nu C D P K 15-A T 1 G 76040$ (AtCPK29), and VvCDPK17-AT3 G20410 (AtCPK9). The second type has a single grape
$C D P K$ gene that corresponds to multiple Arabidopsis genes, including $V v C D P K 3-A T 2 G 17890$ (AtCPK16)/ AT4G36070 (AtCPK18)/ AT5G66210 (AtCPK28), $V v C D$ PK11-AT2G41860 (AtCPK14)/ AT3G57530 (AtCPK32), VvCDPK12-AT1G61950 (AtCPK19)/ AT3G20410 (AtCPK 9)/ AT4G04695 (AtCPK31)/ AT4G21940 (AtCPK15), and VvCDPK14-AT1G74740 (AtCPK30)/ AT1G18890 (AtCP K10). These results provide insights that will assist in prediction of the functions of grape CDPKs.

\section{Phylogenetic analysis of the grape CDPK genes}

To investigate the evolutionary relationships and functional associations, we constructed a neighbor-joining tree using the full-length amino acid sequences of CDPKs from grape, Arabidopsis, rice, maize, and poplar (Fig. 4, Additional file 2). The phylogenetic analysis indicated that nineteen $V v C D P K s$ can be divided into four groups. Of the four groups, three groups appear to be well-defined, except Group IV, according to the distributions of branches. Most of the CDPK genes from monocots (rice and maize) clustered into one sub-branch, as

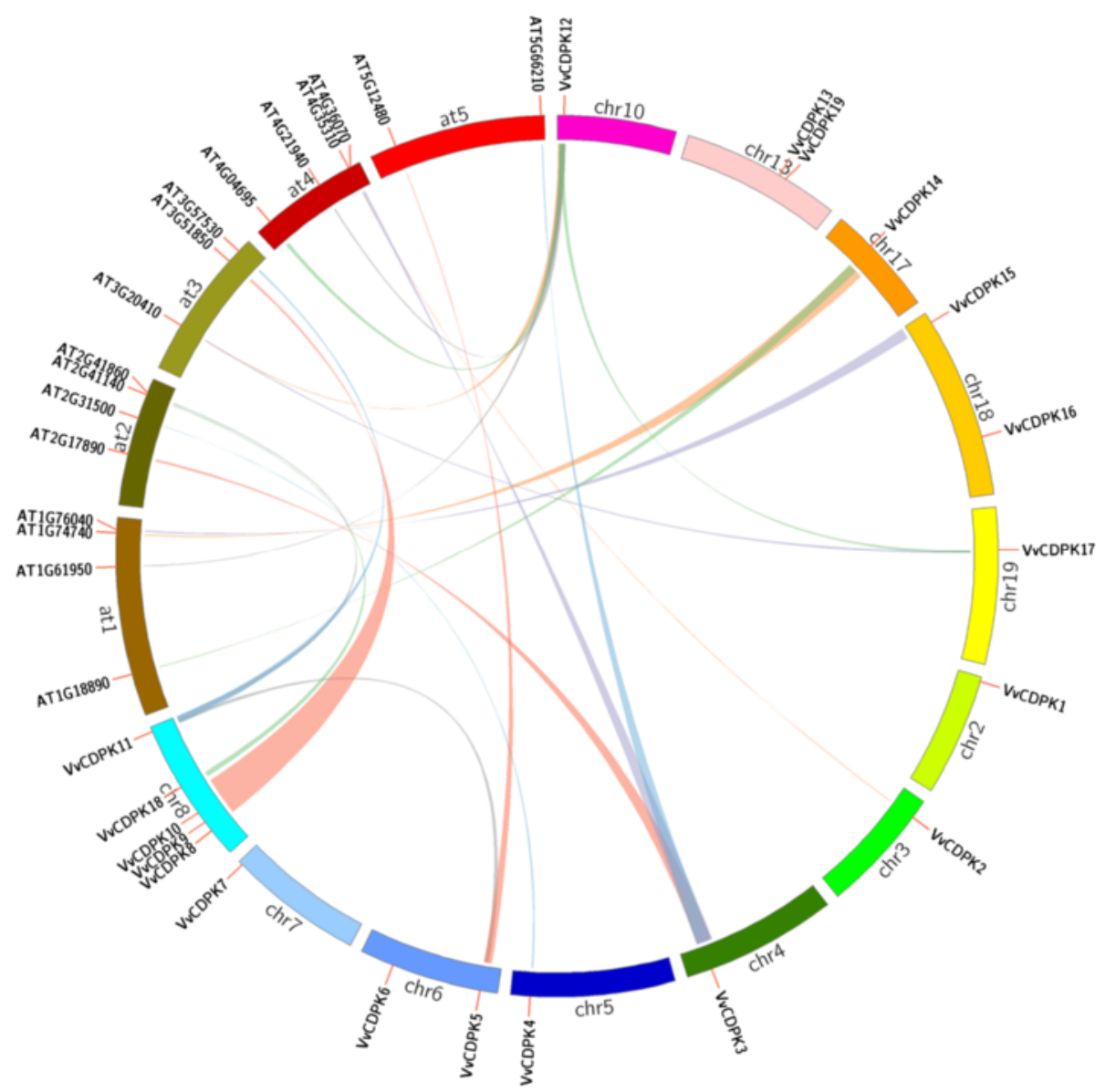

Fig. 3 Segmental duplication of grape CDPK genes and synteny analysis of grape and Arabidopsis CDPK genes. Chromosomes of $V$. vinifera and Arabidopsis are shown in different colors and in circular form. The approximate positions of the AtCDPK and VVCDPK genes are marked with a short red line on the circle. Colored curves denote the syntenic relationships between grape and Arabidopsis CDPK genes 


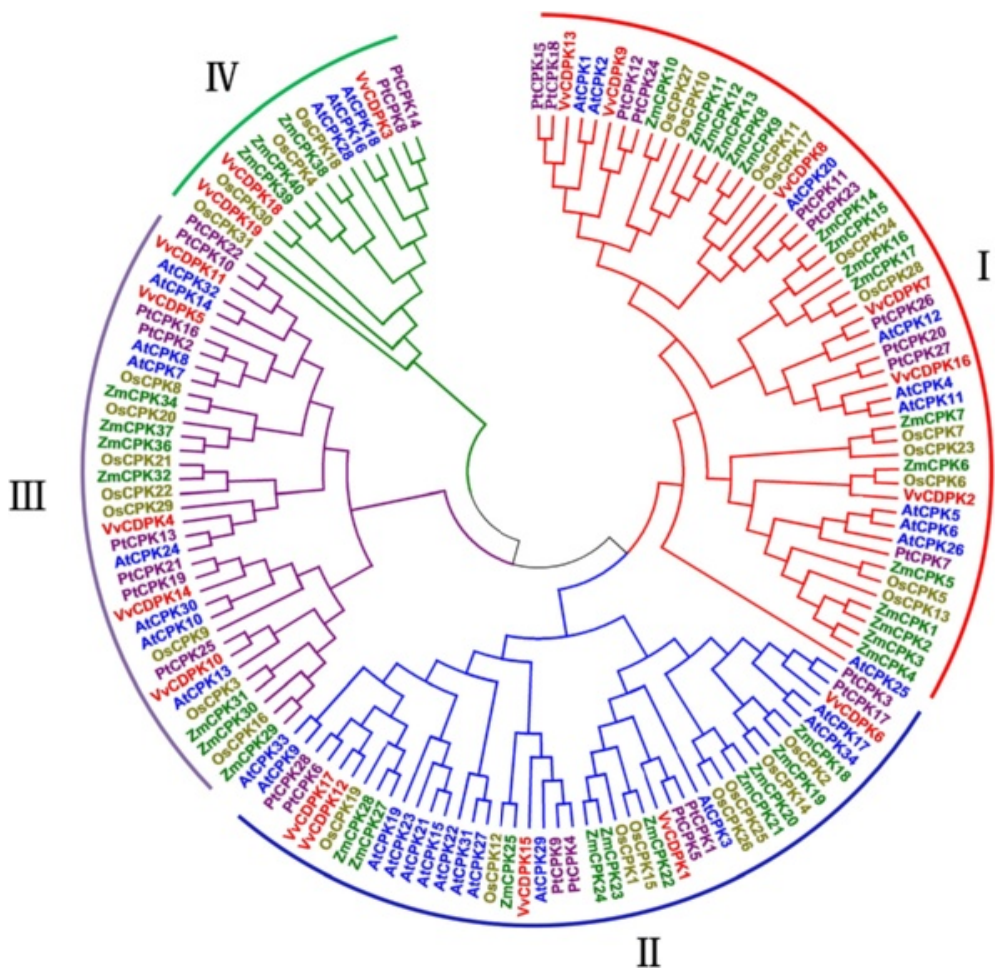

Fig. 4 Phylogenetic analysis of grape CDPK genes. The full-length amino acid sequences of CDPK genes from grape ( $\mathrm{V}$, red), Arabidopsis (At, blue), Populus trichocarpa (Pt, purple), rice (Os, brown) and maize (Zm, green) were aligned by ClustalX and the phylogenetic tree was constructed using the Neighbor-Joining method with 1000 bootstrap replicates by MEGA 5.0. The four subgroups are marked with distinct colors and denoted by Roman numerals

did the CDPK genes from the eudicots (Arabidopsis, poplar and grape). Also, grape CDPK genes clustered more often with poplar CDPK genes than with Arabidopsis CDPK genes. For the four Groups of $V \nu C D P K s$, Group I contains six $V \nu C D P K$ genes. Five of the six genes have homologs in Arabidopsis or poplar, or both, except $V v C D P K 2$, which clustered with ZmCPK6 and OsCPK6. Group II contains five grape CDPK genes and clustered into two subgroups. Among these genes, $V \nu C D P K 12$ and $V \nu C D P K 17$ show high sequence similarity with each other. Group III contains five grape CDPK genes and Group IV, the smallest group, contains three grape $C D P K$ genes. These phylogenetic relationships suggest evolutionary conservation of the basal architecture of the CDPK family.

\section{Subcellular localization of grape CDPKs}

Most CDPKs possess either N-myristoylation sites, Spalmitoylation sites, or both. These acylation sites are believed to be involved in targeting to membranes [9]. Because those acylation sites are predicted to be present in grape CDPKs (Table 1), to determine if grape CDPKs localize to membranes, we cloned six $V p C D P K s$ from Chinese wild Vitis pseudoreticulata accession Baihe-35-1 and assessed the subcellular localizations of the encoded VpCDPKs by transient expression assays in Arabidopsis protoplasts, using translational fusions to GFP.

As shown in Fig. 5, five genes ( $V p C D P K 2, V p C D P K 3$, $V p C D P K 5, V p C D P K 10$, and $V p C D P K 11)$ expressed $\mathrm{VpC}$ DPK-GFP fusion proteins in transformed Arabidopsis protoplasts. VpCDPK3-GFP, VpCDPK10-GFP, and VpC DPK11-GFP only localized on the plasma membrane. VPCDPK5-GFP localized on the plasma membrane and in the nucleus, and VpCDPK2-GFP localized in the nucleus and cytosol.

Unlike the other CDPKs, VpCDPK9 localized to four places (Fig. 6): (i) VpCDPK9 localized on some kind of plastids that could not be identified. Considering the closest gene in Arabidopsis is AtCPK1, which localizes to lipid bodies and peroxisomes $[19,38]$, the plastids that VpCDPK9-GFP localized in may well include lipid bodies and peroxisomes. (ii) VpCDPK9-GFP localized to the biomembrane system, most likely on the endoplasmic reticulum (ER), as well as on vesicles. (iii) VpCDPK9-GFP also showed extra fluorescence in the cytosol. (iv) We also detected VPCDPK9-GFP in the nucleus. The complexity of VpCDPK9 subcellular localization suggests its functional diversity and variety. 


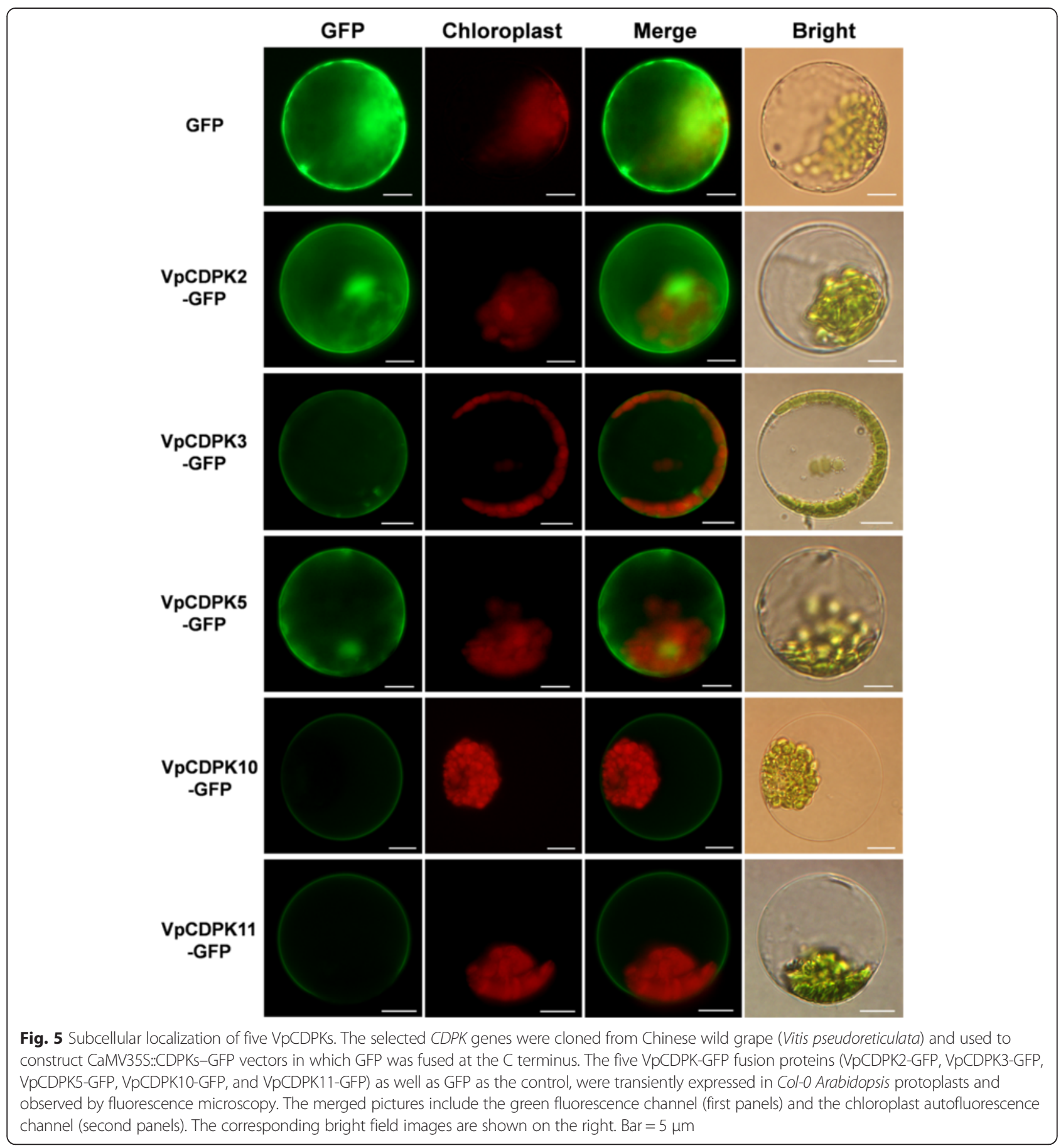

Expression of grape CDPK genes in Chinese wild grape (Vitis pseudoreticulata)

Increasingly evidence shows that plant CDPKs function in responses to biotic and abiotic stress and signal transduction $[9,33]$. To further test how grape CDPK genes respond to various stresses, we measured the expression of grape CDPK genes in Chinese wild grape (Vitis pseudoreticulata) accession Baihe-35-1.

\section{Biotic stress}

CDPKs act as vital sensors and responders in immune signaling [33]. In this study, we used RT-qPCR to perform a time course analysis of the transcript levels of $V p C D P K s$ following inoculation of Chinese wild grape Vitis pseudoreticulata accession Baihe-35-1 with Erysiphe necator, the causal agent of grapevine powdery mildew (Fig. 7-a and Additional file 3). Five of the grape $C D P K$ genes were up-regulated, including 


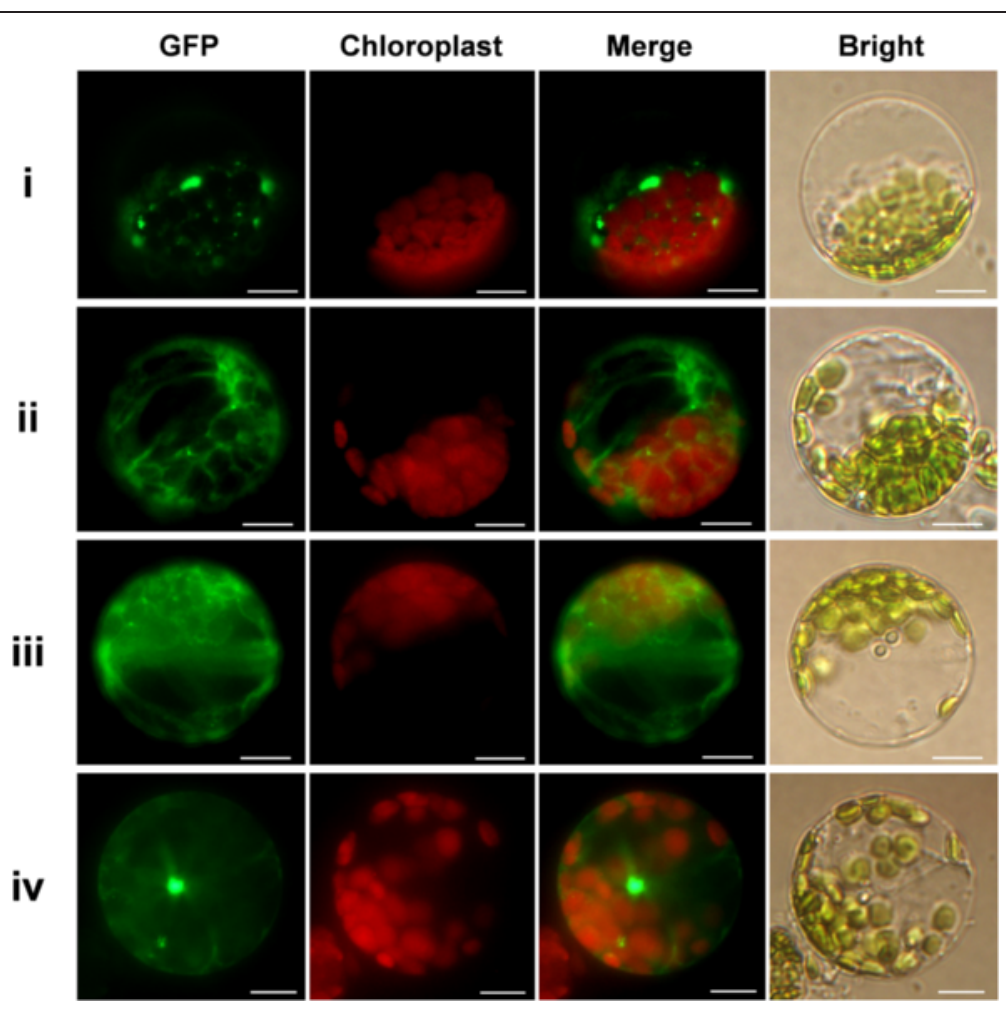

Fig. 6 Subcellular localization of VpCDPK9. The Roman numerals (i-iv) represent the corresponding four subcellular localization patterns of VpCDPK9. VpCDPK9 was cloned from Chinese wild grape (Vitis pseudoreticulata) and used to construct CaMV35S::VpCDPK9-GFP vectors in which GFP was fused at the C terminus. The VpCDPK9-GFP fusion protein, as well as GFP as a control, was transiently expressed in Col-0 Arabidopsis protoplasts and observed by fluorescence microscopy. The merged pictures include the green fluorescence channel (first panel) and the chloroplast autofluorescence channel (second panels). The corresponding bright field images are shown on the right. Bar $=5 \mu \mathrm{m}$

$V p C D P K 6, \quad V p C D P K 9, \quad V p C D P K 14, \quad V p C D P K 16, \quad$ and $V p C D P K 19$, with transcript levels that increased up to 3.0fold $(p<0.05)$ but differed in response time and degree. For example, $V p C D P K 9$ transcript rapidly increased in abundance and reached a peak of 5.3-fold at $96 \mathrm{~h}$ post inoculation (hpi) (Fig. 7-b). Similarly, VpCDPK14 transcript also rapidly increased and remained at high levels (Additional file 3). $V p C D P K 6$ (Additional file 3) and $V p C D P K 19$ (Fig. 7-b) shared similar expression patterns, with a slow increase in transcript levels to a peak and gradual plateau. $V p C D P K 16$ (Fig. 7-b) transcript abundance increased to nearly 3.3-fold, nearly instantly at $72 \mathrm{hpi}$, and remained at high levels. By contrast, several $C D P K$ genes were down-regulated. For instance, $V p C D P K 1$ and $V p C D P K 18$ (Additional file 3) transcript levels decreased to 0.3 - to 0.5 -fold. These results demonstrate that several $C D P K$ genes responded to powdery mildew inoculation in Chinese wild grapevine $V$. pseudoreticulata accession Baihe-35-1, suggesting their vital roles in immune signaling and responses.

\section{Abiotic stress}

Previous work showed that CDPKs participate in abiotic stress responses, including responses to salt and osmotic stress [22, 39] and to temperature stress [33]. In this study, we used $\mathrm{NaCl}$ treatment, and incubation at low or high temperature $\left(4{ }^{\circ} \mathrm{C}\right.$ or $\left.42{ }^{\circ} \mathrm{C}\right)$ to understand how $V p C D P K s$ responded to these abiotic stresses on the transcriptional level. Our expression data show consistency with the expression of CDPK genes in Vitis vinifera [31] and Vitis amurensis [32].

As shown in Fig. 8 (see also Additional files 4, 5, 6), almost all nineteen $C D P K$ genes were up- or downregulated to at least one abiotic stress treatment, but some responded rather slightly. As a whole, the $V p C D P K$ genes responded to $\mathrm{NaCl}$ treatment much more strongly than to temperature stress (Fig. 8-a). $V p C D P K 6$ and $V p C D P K 9$ transcript abundance increased in all three treatments, but none of the $C D P K$ genes showed continuously low transcript levels in all treatments.

After $\mathrm{NaCl}$ treatment (Fig. 8 and Additional file 4), twelve of the nineteen $C D P K$ genes were up-regulated, and six genes ( $V p C D P K 3, V p C D P K 9, V p C D P K 14, V p C$ $D P K 16, V p C D P K 17$, and $V p C D P K 19)$ responded strongly with transcript levels increasing to 5.0- to 16-fold higher than the control sample $(p<0.05) . V p C D P K 9$, the most 

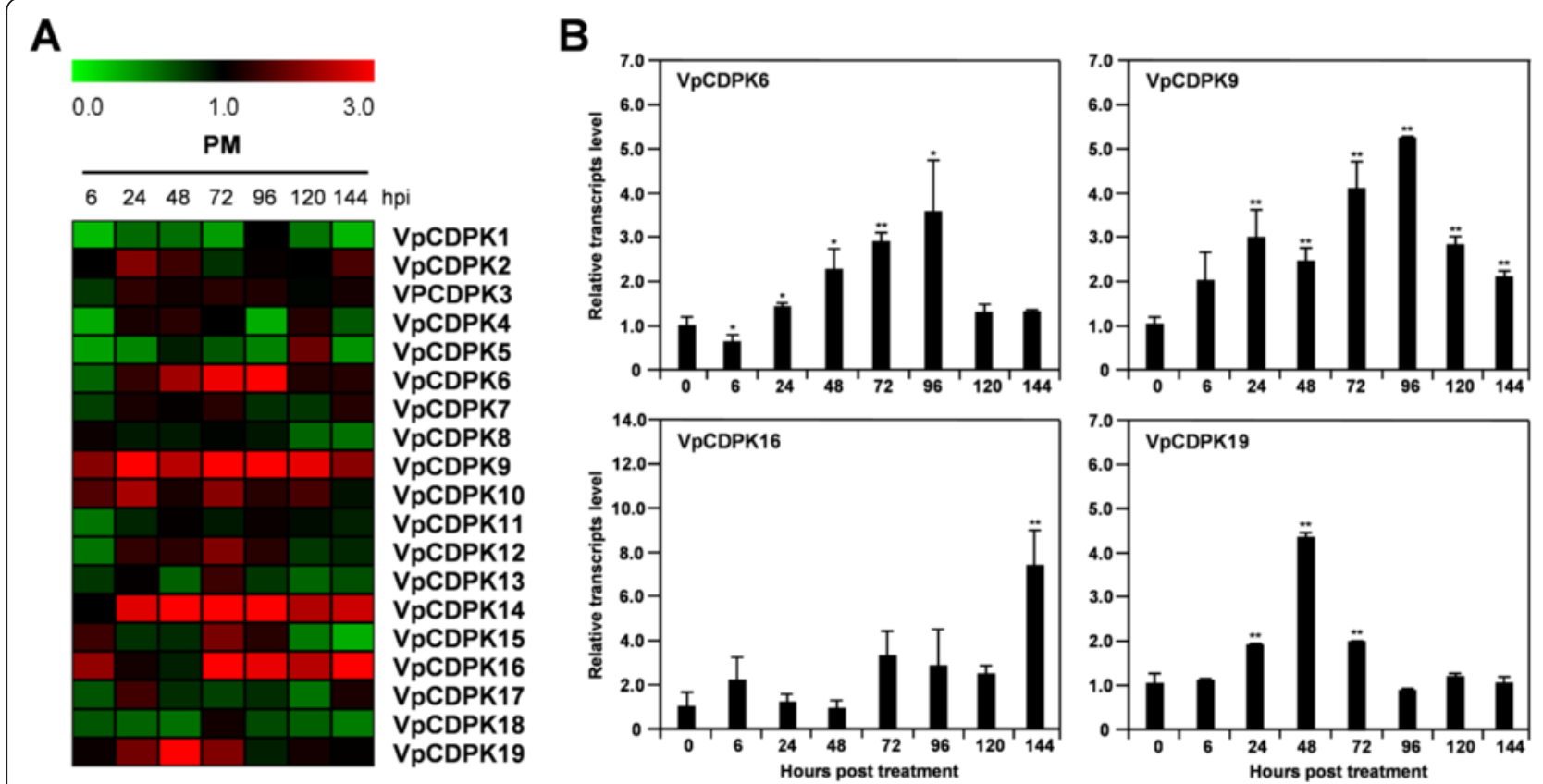

Fig. 7 Expression of CDPK genes in Chinese wild grape (Vitis pseudoreticulata) during powdery mildew infection. Expression was measured by reverse transcription, followed by real-time, quantitative $P C R$, is indicated as fold-change of experimental treatments relative to control samples, and is visualized as heatmaps (A) and histogram (B). Grape Actin1 (GenBank Accession number AY680701) was used as an internal control. The experiments were repeated three times and gave consistent results. Mean values and SDs were obtained from three biological and three technical replicates. (A) Expression profile of VPCDPK gene family under powdery mildew infection. The color scale represents log 2 expression values, with red indicating increased transcript abundance and green indicating decreased transcript abundance. (B) Detailed expression levels of four VpCDPK genes that significantly up-regulated during powdery mildew infection. The data were showed as mean value $\pm \mathrm{SD} .{ }^{*}$ and ${ }^{* *}$ represent statistically significant $(p<0.05)$ or highly significant $(p<0.01)$, respectively

rapidly responding gene, reached a peak of nearly 10fold at $0.5 \mathrm{~h}$ post treatment (hpt) and rapidly decreased to 3.9 -fold at $2 \mathrm{hpt}$, before its transcript levels increased from 3.9-fold to 9.7-fold at $2 \mathrm{~h}$ to $12 \mathrm{hpt}$ (Fig. 8-b). The homologous gene of VpCDPK9 in Vitis amurensis, $\mathrm{VaCPK1}$, was also highly up-regulated under $\mathrm{NaCl}$ treatment [32]. In addition, $V P C D P K 17$ showed a peak of 16.3-fold at $8 \mathrm{hpt}$ and increased transcript abundance of more than 9.0-fold from 2 to 48 hpt (Fig. 8-b), similar with the expression pattern of its homologs, VaCPK9 [32] and $V \nu C P K 17$ [31]. We noted that the margins of treated leaves became dry from the outside to the inside and the plants had dry-dead leaves after $48 \mathrm{hpt}$, which might relate to the rapid down-regulation of $V p C D P K 14$ at 48 hpt. By contrast, VpCDPK5 (Fig. 8-b) and $V p C D P K 8$ (Additional file 4) transcript abundance decreased after $2 \mathrm{hpt}$, indicating their probable negative regulatory roles. The CDPK genes that responded rapidly and strongly most likely participate in the $\mathrm{NaCl}$ stress response.

For $4{ }^{\circ} \mathrm{C}$ treatments (Fig. 8 and Additional file 5), only $V p C D P K 6, V p C D P K 9$ and $V p C D P K 16$ (Fig. 8-a) showed up-regulation $(p<0.05)$. $V p C D P K 6$ was rapidly upregulated, reached a peak of 12.4 -fold at $2 \mathrm{hpt}$, and then declined to lower than 2.0- fold (Fig. 8-b). Moreover, $V p C D P K 16$ had a peak of 7.7 -fold at 8 hpt but then fluctuated around 1.0-fold during the rest of the treatment time (Fig. 8-b). The VpCDPK genes with decreased transcript abundance might not participate in the signaling response to cold stress but their low activities might result in pervasive down-regulation of transcription. However, $V p C D P K 8$ (Fig. 8-b) was an exception, as its transcript levels decreased rapidly at $2 \mathrm{hpt}$ and remained at a steady low level. Intriguingly, $\mathrm{VaCPK} 20$, as the homolog of $V p C D P K 8$ in Vitis amurensis, showed significant up-regulation under $10{ }^{\circ} \mathrm{C}$ treatment [32].

We also measured the transcript levels of $V p C D P K$ genes in response to $42{ }^{\circ} \mathrm{C}$ treatment. We found that five $C D P K$ genes (VpCDPK4, VpCDPK6, VpCDPK9, VpCDPK10, and $V p C D P K 19)$ significantly responded to $42{ }^{\circ} \mathrm{C}$ treatment (the peaks are over than 3.0- fold, $p<0.05$ ); of these, $V p C D P K 6$ (Fig. 8-b) had a strong response at $2 \mathrm{hpt}$, with its transcript abundance increasing to 14.6-fold and remaining at a high level. The transcript abundance of $V p C D P K 9$ (Fig. 8-b) and $V p C D P K 19$ (Additional file 6) increased, similar to $V p C D P K 6$ (Fig. 8-b), although $V p C D P K 6$ transcripts were almost undetectable at $48 \mathrm{hpt}$. In particular, $V p C D P K 4$ (Fig. 8-b) responded to $42{ }^{\circ} \mathrm{C}$ treatment rapidly and 
A
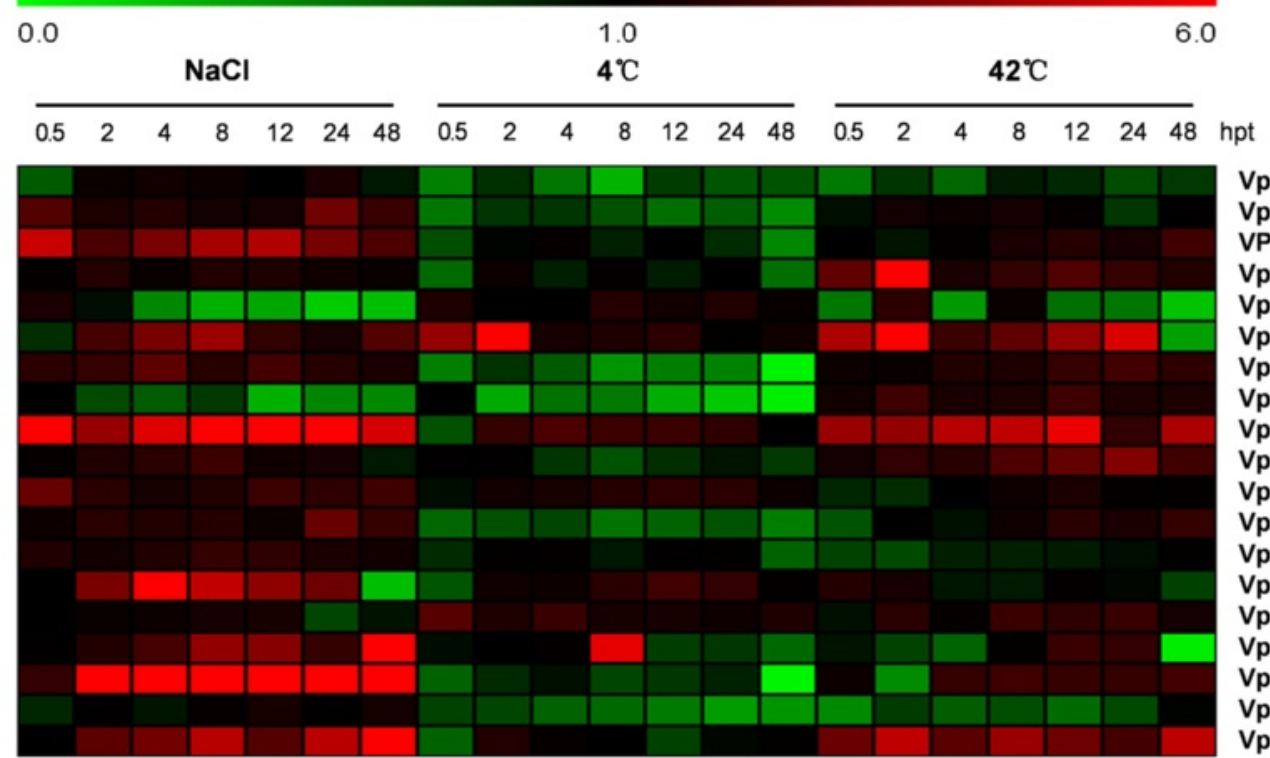

VpCDPK1

VpCDPK2

VPCDPK3

VpCDPK4

VpCDPK5

VpCDPK6

VpCDPK7

VpCDPK8

VpCDPK9

VpCDPK10

VpCDPK11

VpCDPK12

VpCDPK13

VpCDPK14

VpCDPK15

VpCDPK16

VpCDPK17

VpCDPK18

VpCDPK19

\section{B}
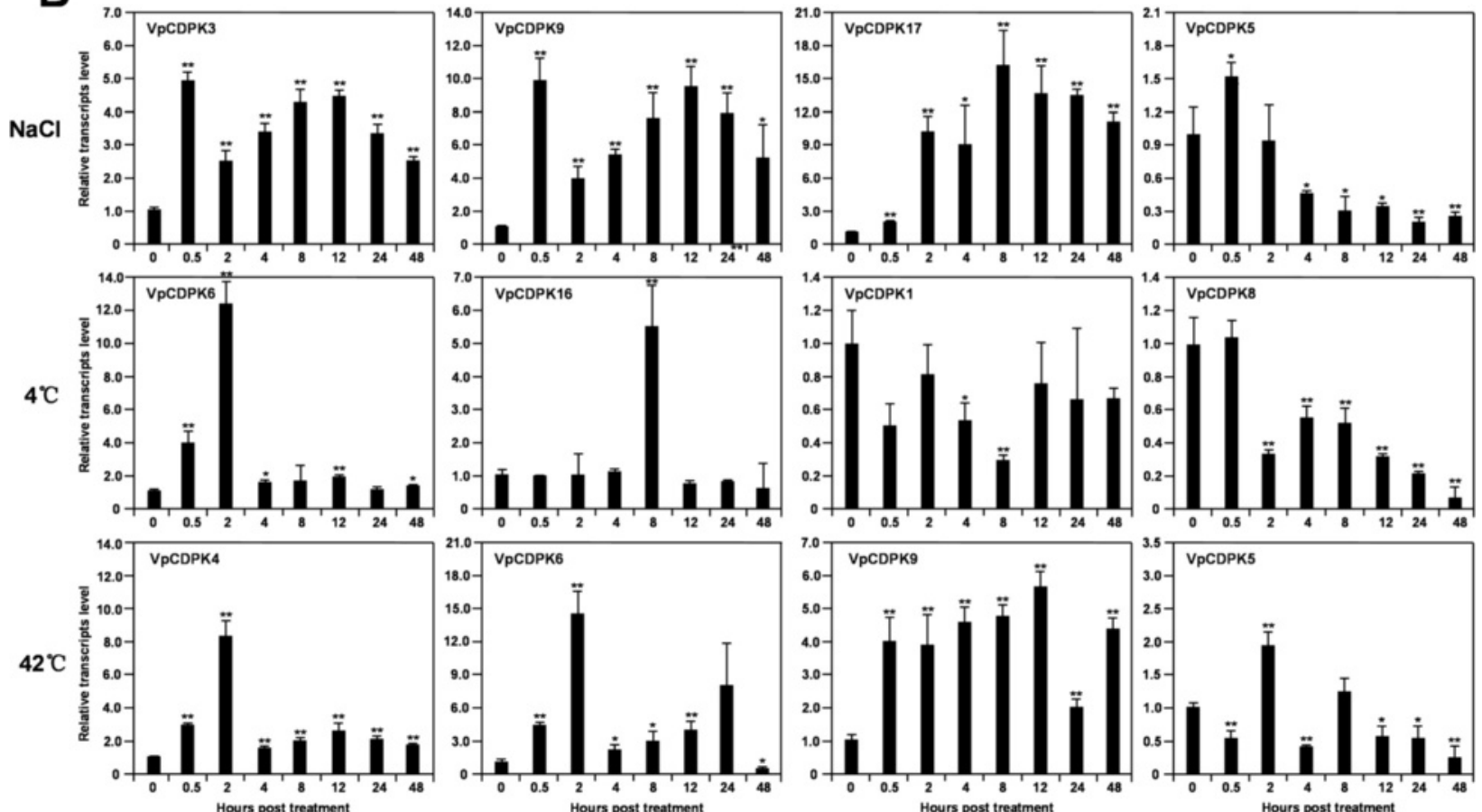

Fig. 8 Expression of CDPK genes in Chinese wild grape (Vitis pseudoreticulata) under salt and temperature stress treatments. Expression was measured by reverse transcription, followed by real-time, quantitative $P C R$, is indicated as fold-change of experimental treatments relative to control samples, and is visualized as heatmaps a and histogram b. Grape Actin1 (GenBank Accession number AY680701) was used as an internal control. The experiments were repeated three times and gave consistent results. Mean values and SDs were obtained from three biological and three technical replicates. a Expression profile of VPCDPK gene family under $\mathrm{NaCl}, 4^{\circ} \mathrm{C}$, and $42{ }^{\circ} \mathrm{C}$ treatments. The color scale represents log 2 expression values, with red indicating increased transcript abundance and green indicating decreased transcript abundance. $\mathbf{b}$ Detailed expression levels of four VpCDPK genes showed unusual expression patterns under $\mathrm{NaCl}, 4^{\circ} \mathrm{C}$, and $42^{\circ} \mathrm{C}$ treatments. The data were showed as mean value $\pm S D .{ }^{*}$ and ${ }^{* *}$ represent statistically significant $(p<0.05)$ or highly significant $(p<0.01)$, respectively 


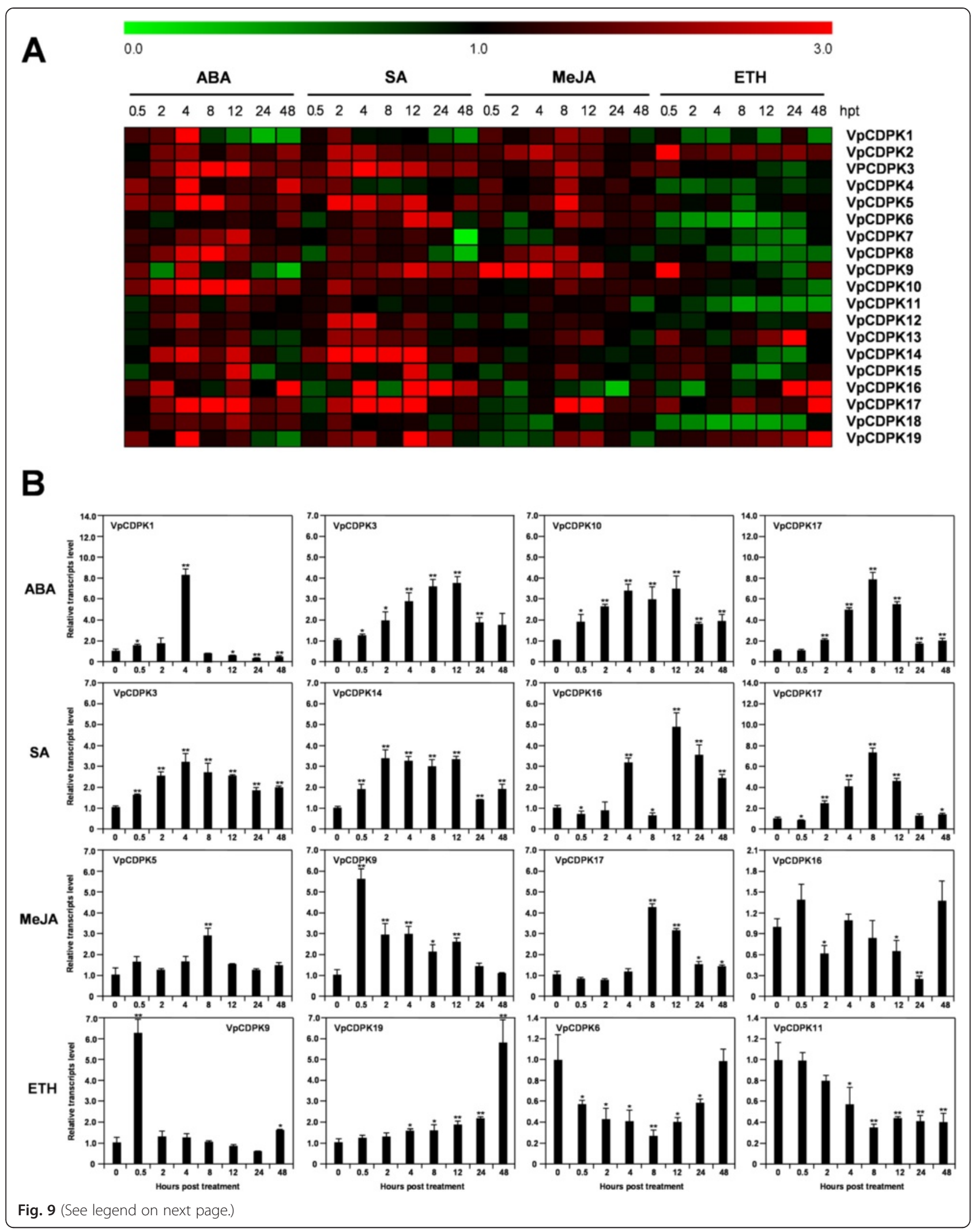


(See figure on previous page.)

Fig. 9 Expression of CDPK genes in Chinese wild grape (Vitis pseudoreticulata) in response to treatment with plant hormones. Expression was measured by reverse transcription, followed by real-time, quantitative $P C R$, is indicated as fold-change of experimental treatments relative to control samples, and is visualized as heatmaps (a) and histogram (b). Grape Actin 1 (GenBank Accession number AY680701) was used as an internal control. The experiments were repeated three times and gave consistent results. Mean values and SDs were obtained from three biological and three technical replicates. a Expression profile of VPCDPK gene family under ABA, SA, MeJA, and ethylene (ETH) treatments. The color scale represents log2 expression values, with red indicating increased transcript abundance and green indicating decreased transcript abundance. $\mathbf{b}$ Detailed expression levels of four VpCDPK genes showed unusual expression patterns under ABA, SA, MeJA, and ethylene (ETH) treatments. The data were showed as mean value $\pm S D$. * and ${ }^{* *}$ represent statistically significant $(p<0.05)$ or highly significant

$(p<0.01)$, respectively

intensely, reaching a peak of more than 8.3-fold and then returning to nearly normal transcript levels at $4 \mathrm{hpt}$. Taken together, our data showed that expression of $V p C D P K s$ responded to low and high temperatures $\left(4{ }^{\circ} \mathrm{C}\right.$ and $\left.42{ }^{\circ} \mathrm{C}\right)$ suggest that the $V p C D P K s$ may play key roles in the response to temperature stress.

\section{Hormone treatment}

Plant hormones such as ABA, SA, MeJA, and ethylene have well-established roles in modulating plant signaling networks [40]. In this study, hormone treatments resulted in a wide variety of changes in the transcript levels of $V p C D P K$ genes (Fig. 9 and Additional files 7, 8, $9,10)$.

An increasing body of evidence has shown that CDPKs regulate ABA-mediated signal transduction in plants [22, 23, 41]. Our experimental data suggest that 16 of the $19 \mathrm{VpCDPK}$ genes respond to ABA treatment (Fig. 9 and Additional file 7). Among them, eight $V p C D P K$ genes (VpCDPK1, 3, 4, 5, 10, 16, 17, 19) showed significant up-regulation in response to $\mathrm{ABA}$ (the peaks are over than 3.0- fold, $p<0.05$ ). However, $V p C D P K 1$ (Fig. 9-b) transcript levels rapidly increased up to 8.3 -fold at $4 \mathrm{hpt}$ and then decreased to lower than 0.3 -fold at $8 \mathrm{hpt}$. Interestingly, the majority of the responsive CDPK genes showed a similar pattern, with their transcript abundance increasing rapidly to a peak at $4 \mathrm{hpt}$ and remained at high levels until $12 \mathrm{hpt}$.

Similar to the ABA response, 13 of the $19 \mathrm{VpCDPK}$ genes also showed positive regulation under SA treatment (Fig. 9 and Additional file 8). Six genes ( $V p C D P K 3$, $5,14,16,17,19)$ were significantly up-regulated (the peaks are over than 3.0-fold, $p<0.05$ ), exhibiting a steady or gradual increase from 2 to 12 or 2 to $24 \mathrm{hpt}$. Interestingly, the expression of $V p C D P K 6, V p C D P K 9$, $V p C D P K 14$, and $V p C D P K 16$ was also induced after inoculation with powdery mildew pathogen (Fig. 7). For instance, $V p C D P K 9$ showed positive regulation by inoculation of powdery mildew and peaked ( 5.5 -fold) at 96 hpi (Fig. 7-b), while under SA treatment, $V p C D P K 9$ was also up-regulated and peaked $(\sim 2.8$-fold $)$ at $12 \mathrm{hpt}$ (Additional file 8). The expression pattern after SA treatment and powdery mildew inoculation, suggests the vital roles of grape $C D P K$ genes in immune signaling.

In contrast with $\mathrm{ABA}$ and $\mathrm{SA}$, the $V p C D P K s$ showed more limited responses to MeJA treatment. A total of ten genes $(V p C D P K 1,2,3,4,5,6,8,9,17,19)$ were upregulated; and among them, only three $V p C D P K$ genes $(V p C D P K 5,9,17)$ showed distinct up-regulation (the peaks are over than 3.0-fold, $p<0.05$ ) (Fig. 9 and Additional file 9). In particular, their transcript abundance peaked at 4 or 8 hpt, except for VpCDPK9 (Fig. 9-b), which strongly and rapidly responded to the treatment from 0.5 to $12 \mathrm{hpt}$ with MeJA and peaked at $0.5 \mathrm{hpt}$. That might indicate its functional significance in the MeJA signaling pathway.

The expression changes caused by ethylene treatment (Fig. 9 and Additional file 10) were distinct from those caused by the other hormone treatments. For ethylene treatment, only a handful of $V p C D P K$ genes $(V p C D P K 2$, $9,13,16,17,19)$ were up-regulated $(p<0.05)$, while more genes showed down-regulation. For example, two up-regulated genes, $V p C D P K 2$ (Additional file 10) and $\operatorname{VpCDPK9}$ (Fig. 9-b), their transcript abundance increased rapidly at $0.5 \mathrm{hpt}$, but then increased only slightly or returned to normal transcript levels, respectively. Chinese wild grapevine Baihe-35-1 seems to be very sensitive to ethylene treatment and almost all the leaves of Baihe-35-1 plants fell at $48 \mathrm{hpt}$, so we had to use the fallen leaves for RNA extraction. $V p C D P K 6$, $V p C D P K 7, V p C D P K 8, V p C D P K 11$, and $V p C D P K 18$ had decreased transcript abundance lower than 0.5- fold during the treatment time, suggesting their potential negative regulatory functions in plant responses to ethylene signaling.

\section{Discussion}

\section{Identification of Grape CDPK genes}

Previously, Chen [31] investigated the CDPK gene family in grapevine and identified 17 members, which were predicted to have the typical CDPK gene structure with a Ser/Thr protein kinase domain and four EF-hands. Considering the complexity and variability in the calmodulin-like domain, we performed BLAST-P searches 
in NCBI using the putative grape CDPK genes predicted by Pkinase (PF00069) and EF-hand_7 (PF13499) HMM profiles, and identified the previously discovered 17 CDPKs and two more members (Fig. 1). Compared with Arabidopsis CDPKs [9] and rice CDPKs [10], we found that GSVIVT01025745001 (LOC100246588 in NCBI, designated here as $V v C D P K 18)$ possesses a similar domain organization to AtCPK25 and OsCPK30, which are predicted to have no EF-hand with default parameters in Pfam but are predicted to have EF-hands in PROSITE and InterPro. In addition, the phylogenetic tree showing that GSVIVT01025745001 ( $V v C D P K 18)$ and OsCPK30 are homologous genes provides more evidence (Fig. 4). Therefore, we considered GSVIVT01025745001 ( $V v C$ $D P K 18)$ as members of the grape $C D P K$ gene family and the same with GSVIVT01027353001 (LOC100250591 in $\mathrm{NCBI}$, designated here as $V v C D P K 19)$. It is worth to mention that a homolog of $V v C D P K 19$ in Vitis amurensis, named as VaCPK25, has been identified as a $C D P K$ gene [32]. Both of these two genes (VpCDPK19 and $\mathrm{VaCPK} 25)$ are same in gene length and phylogenetic relationship. Consider that, $V p C D P K 19$ can also be identified as a $C D P K$ gene. These two new members of the grape $C D P K$ gene family were designated $V v C D P K 18$ and $V \nu C D P K 19$ on the basis of their chromosomal locations.

\section{Structural characteristics of grape CDPK genes}

The structural conservation and divergence of grape $C D P K$ genes led to gene family expansion and functional conservation or differentiation. Structural characteristics such as acylation sites and intron-exon structures show the details of gene family expansion and divergence.

Most of CDPKs possess acylation sites, including Nmyristoylation sites and S-palmitoylation sites, which are generally believed to function in membrane targeting [9]. In Arabidopsis, 21 AtCPKs have both myristoylation and palmitoylation sites and all of them target exclusively to membranes, especially to the plasma membrane, although several have not been determined [33]. Among our identified nineteen grape CDPK genes, six contain both myristoylation and palmitoylation sites, nine contain only palmitoylation sites, two contain only myristoylation sites, and two do not contain any acylation site (Table 1). We tested the subcellular localization of six VpCDPK proteins. VpCDPK2, VpCDPK5, VpCDPK10, and VpCDPK11 have palmitoylation sites while VpCDPK3 has a myristoylation site (Table 1 ) and they all localized on the plasma membrane (Fig. 5), consistent with results in Arabidopsis [33].

Three pivotal mechanisms contribute to gene family evolution and expansion: exon/intron gain or loss, exonization/ pseudo-exonization, and insertion /deletion [42]. Previous work reported that grape CDPK genes have a single origin and can be dated back to green algae, before plants colonized the land [43]. For the four groups in Fig. 2, Group IV was the earliest one that expanded from the evolutionary branch [31]. As a result, it has the longest evolutionary history, leading to complex intron-exon organization. Both Group II and Group III originated from Group I in evolutionary history, and show similar structural divergence. All of the members of Group II contain one more exon and a phase- 2 intron at the sixth position, indicating that there was an intron insertion in the last exon, which contributed to the generation of Group II and expansion of the $V \nu C D P K$ family. Group III can be separated into two subgroups in evolutionary history, as reflected in their intron-exon structures. One subgroup was similar to Group I in intron-exon structures and the other one contains one more exon and a phase- 0 intron at the first position, suggesting that there was an intron insertion in the first exon. Our result is consistent with the previous work that can be reflected in the origin and evolutionary history of $V v C P K s$ [31]. The intron-exon divergence was closely related to the evolutionary relationship of the grape CDPK family and might result in functional diversity.

\section{Evolutionary relationships of grape CDPK genes}

To study the evolutionary relationships among different $V \nu C D P K$ genes and the history of the $V \nu C D P K$ gene family, as well as to further study their gene function, we investigated gene duplication events, syntenic regions, and phylogenetic relationships among the $V \nu C D P K$ genes.

Segmental duplications and tandem duplications are the main mechanisms leading to gene family expansion [44]. These processes may lead to functional redundancy, sub-functionalization and neo-functionalization. No tandem duplication involving $V \nu C D P K$ genes was discovered in the grapevine genome, but two segmental duplications were found, $V \nu C D P K 5-V v C D P K 11$ and $V \nu C$ $D P K 12-V v C D P K 17$ (Fig. 3, Additional file 1). Previously, Chen identified three segmental duplications [31]. Except for $V \nu C D P K 5-V \nu C D P K 11$ and $V \nu C D P K 12-V \nu C D$ $P K 17$, they also identified triplet-duplicated genes, $V \nu C P K 8-V \nu C P K 9-V \nu C P K 13$. However, the method they used was not clearly presented, so we cannot follow their method. Anyway, both of Chen and us indeed identified the two duplicated gene pairs, $V \nu C D P K 5-V \nu C D P K 11$ and $V \nu C D P K 12-V v C D P K 17$. These two duplicated gene pairs are quite similar in gene length, acylation sites, and intron-exon organization (Table 1, Fig. 2). Furthermore, they possess the closest phylogenetic relationship among grape CDPK genes (Fig. 4). However, despite the fact $V \nu C D P K 12$ and $V v C D P K 17$ are phylogenetically closest among CDPKs from other species, these two genes have 
different syntenic genes in Arabidopsis (Fig. 3, Additional file 1), which means the duplication events happened before the evolutionary divergence of Arabidopsis and grapevine. Both $V v C D P K 5$ and $V \nu C D P K 11$ showed closer phylogenetic relationships with Arabidopsis CDPK genes than that with each other, indicating that this duplication event might also have happened before the divergence of Arabidopsis and grapevine. From what has been discussed above, $V \nu C D P K 5-V v C D P K 11$ and $V \nu C D P K 12-V \nu C D P K 17$ likely have different functions but still possess potential functional connections and similarities.

Comparative genomics approaches structure genomes into syntenic blocks that exhibit conserved features across the genomes [45]. The synteny analysis provides evolutionary and functional connections between grape and Arabidopsis syntenic genes. Furthermore, a large number of syntenic relationships suggest that some of the grape CDPK genes arose before the divergence of the Arabidopsis and grapevine lineages. Ten grape CDPK genes were found to have syntenic relationships with Arabidopsis genes (Fig. 3) All of these VvCDPK genes show close phylogenetic relationships with the corresponding AtCDPK genes (Fig. 4), suggesting their potential functional similarities. Interestingly, all of the grape CDPK genes in Group III (Fig. 4) have syntenic genes in Arabidopsis, suggesting that Group III CDPK genes are relatively conserved over evolutionary history.

Analysis of the phylogenetic tree revealed that CDPK homologs among several monocots and eudicots clustered into four distinct groups, which correspond to the clades formerly identified in green plants [43]. Previously, Hamel [43] examined the CDPK families from green algae to land plants to demonstrate that CDPK families are conserved among land plants, whereas CDPKs from green algae have continued to evolve independently. Supporting this, our data suggest that CDPKs from monocots and eudicots cluster separately (Fig. 4) and evolutionary relationships of the four clades are relatively conserved. The previous work [31] also constructed a phylogenetic tree that gives the consistent results and conclusions.

\section{Functional prediction of grape CDPK genes by comparison with Arabidopsis}

Comparative genomics provides an effective way to understand the structure and function of genomes by translating knowledge gained from model species to the species of interest. Combining synteny analysis with phylogenetic analysis provides new insights for further investigating the functions of the grape CDPK genes by comparing orthologous genes between two species, in this case, between grapevine and Arabidopsis. Our study provided substantial evidence to help predict the functional conservation or divergence of $C D P K$ genes between grapevine and Arabidopsis.
Chinese wild grape (Vitis pseudoreticulata) accession Baihe-35-1, compared with the sequenced Vitis vinifera, reported to have remarkable resistance to both biotic and abiotic stress [29, 30]. In addition, Vitis amurensis is also a wild grapevine species with a high level of resistance to multiple stresses [32]. Our expression profile of $V p C D P K$ genes showed consistency with that reported in Vitis vinifera [31] and Vitis amurensis [32]. For example, the homologous $C D P K$ genes, $V p C D P K 3 / V v C P$ $K 3 / \mathrm{VaCPK} 16$ and $V p C D P K 17 / \mathrm{V} \nu \mathrm{CDPK} 17 / \mathrm{VaCPK} 17$ showed distinct up-regulation under salt stress treatment [31, 32] (Fig. 8-b). However, there are also some differences on genes expression, e.g., $\mathrm{VaCPKs}$ respond to cold stress much significantly than $V p C D P K s$, probably because of Vitis amurensis shows strong capacity of cold-resistance.

Two duplicated gene pairs, $V p C D P K 5 / V p C D P K 11$ and $V p C D P K 12 / V p C D P K 17$, as discussed above, provide experimental evidence to support our predictions and views. $V p C D P K 5$ and $V p C D P K 11$ each have particular syntenic genes in Arabidopsis showing a complicated evolutionary history. In addition, the experimental data showed that these two genes have distinct expression patterns under a range of treatments. For example, $V p C D P K 5$ was obviously up-regulated under hormone treatments (except ethylene), while $V p C D P K 11$ was only slightly up-regulated under ABA treatment (Fig. 9 and Additional files 7, 8, 9, 10). Also, for abiotic stress treatments, $V p C D P K 5$ was down-regulated under $\mathrm{NaCl}$ and $42{ }^{\circ} \mathrm{C}$ treatments, but $V p C D P K 11$ transcripts remained at constant levels (Fig. 8 and Additional files 4, 6). Furthermore, subcellular localization analysis demonstrated that VpCDPK5-GFP localized on plasma membrane and in the nucleus, but VPCDPK11-GFP only localized on plasma membrane (Fig. 5). The aforementioned information indicated that $V p C D P K 5$ and $V p C D$ PK11 might well have undergone neo-functionalization. Moreover, $V v C D P K 11$ in $V$. vinifera cv. Corvina expressed in almost all of the organs, whereas $V \nu C D P K 5$ only expressed in pollen [31]. However, we tested VpCDPK5 in the Chinese wild grape $V$. pseudoreticulata responded to hormone treatments (Fig. 9) in leaves, suggesting that $V p C D P K 5$ was not only transcribed in pollen. For $V p C D P K 11$, its low expression under multiple treatments (Figs. 7, 8, 9) and high expression in almost all tissues [31] indicates that its function most likely relates to housekeeping genes. The other duplicated gene pair, $V p C D P K 12$ and $V p C D P K 17$ were quite different. The transcript levels of these two genes showed similar tendencies under abiotic stress, ABA and SA treatments (Figs. 8, 9 and Additional files 4, 5, 6, 7, 8), however, $V p C D P K 17$ seemed to respond much strongly than $V p C D P K 12$. Based on Chen [31], $V v C D P K 17$ had high transcript levels in almost all tissues while $V \nu C D P K 12$ had low transcript levels. 
These data suggest that the duplicated gene pair $V p C D P K 12 / V p C D P K 17$ probably have undergone subfunctionalization and $V p C D P K 12$ tends to be nonfunctional.

It is worth noting that $V p C D P K 9$ is unique in the grape $C D P K$ family. For one thing, it showed an unusual expression pattern under various treatments, being strongly up-regulated, except for $4{ }^{\circ} \mathrm{C}$ treatment (Fig. 7, 8 , 9). Its transcript abundance increased to a high level during most or all of the treatment (Figs. 7, 8, 9). Subcellular localization also demonstrated its considerable differences with the other grape CDPKs we examined. The other VpCDPKs have only one pattern of localization (Fig. 5), while VpCDPK9 shows four patterns, including in some kind of plastids, in biomembranes, in the cytosol, or in the nucleus (Fig. 6). Testing why VpCDPK9 has four patterns of localization, and the relationship between its localization and function, will require more experimental data. The genes that are phylogenetically close to $V p C D P K 9$ in Arabidopsis are AtCPK1 and AtCPK2 (Fig. 4). VpCDPK9 has structural and functional commonalities with AtCPK1. For example, AtCPK1 expression is rapidly induced by fungal elicitors and loss-of-function mutants of $A t C P K 1$ exhibit higher susceptibility to pathogen infection [19]. Ectopic expression of AtCPK1 enhanced NADPH oxidase activity and the oxidative burst in tomato protoplasts [18]. Cold-stress can also induce $A t C P K 1$ transcripts via phosphoprotein signals [46]. Our data also showed that $V p C D P K 9$ not only responded to powdery mildew infection and abiotic stress, but also responded to hormone treatments (Figs. 7, 8, 9). These results are consistent with the expression profile of AtCPK1. Future work will be directed toward identification of potential defense components that may be directly or indirectly regulated by VpCDPK9 in grapevine Baihe-35-1.

$V p C D P K 2$, whose Arabidopsis homolog is AtCPK5, is found in the chromosomal region syntenic with AtCPK5, and plays positive regulatory roles in various treatments (Figs. 7, 8, 9). Results on AtCPK5 show high consistency with our results on $V p C D P K 2$. (i) AtCPK5 was reported to localize to the plasma membrane and the nucleus [47, 48], consistent with our data showing that $V p C D P K 2$ also localized to the plasma membrane and nucleus (Fig. 5). (ii) $A t C P K 5$ can localize to the nucleus where it interacts with and phosphorylates WRKY8, 28, and 48 to activate defense genes [48], while VpCDPK2 also localized in the nucleus (Fig. 5), suggesting it has similar functions with AtCPK5. (iii) AtCPK5 also phosphorylates $\mathrm{RBOHD}$ in vivo, resulting in $\mathrm{H}_{2} \mathrm{O}_{2}$ production and leading to cell death [21, 49], involving in immune signaling. $V p C D P K 2$ responded to powdery mildew inoculation with up-regulated transcript levels (Fig. 7), indicating its potential function in immune signaling and that $V p C D P K 2$ most likely participates in the analogous pathways to those mentioned above for Arabidopsis. These structural and locational similarities provide indications of functional consistency.

$V p C D P K 10$ has a syntenic gene in Arabidopsis, AtCPK13. Further analysis provides valuable comparisons of these two genes and helps predict the function of $V p C D P K 10$. In our study, $V p C D P K 10$ localized on the plasma membrane (Fig. 5), and AtCPK13 was also reported to localize on the plasma membrane [50]. $V p C D P K 10$ expression did not respond to stress treatments but was distinctly up-regulated in response to ABA treatment (Fig. 8). AtCPK13 specifically inhibits KAT1 and KAT2 shaker channels [50], which participate in ABA-induced stomatal movements by affecting CDPK phosphorylation [41]. These insights indicate that $V p C D P K 10$ plays important roles in $\mathrm{ABA}-$ and $\mathrm{Ca}^{2+}$-mediated stomatal regulation.

Another intriguing gene, $V p C D P K 16$, is phylogenetically close to $A t C P K 4$ and $A t C P K 11$ (Fig. 4), which are activated by ABA and phosphorylate the C-terminus of ACS6 in ethylene biosynthesis [28]. Meanwhile, AtCPK4 and AtCPK11 can phosphorylate the ABA-responsive transcription factors ABF1 and ABF4 and lead to salt insensitivity in seed germination and decrease tolerance of seedlings to salt stress [22]. Sustained AtCPK4 and AtCPK11 activation directly phosphorylated WRKY transcription factors involved in immune signaling [51]. Expression patterns suggest that $V p C D P K 16$ responded to powdery mildew inoculation as well as $\mathrm{NaCl}, \mathrm{ABA}$, and SA treatments (Figs. 7, 8, 9), showing high functional consistency with AtCPK4 and AtCPK11. This evidence indicates that $V p C D P K 16$ might participate in similar pathways as AtCPK4 and AtCPK11 in Arabidopsis.

In addition to stress, CDPK genes also play important roles in growth and development process. On this regard, plant hormones are mainly responsible. For instance, several plant hormones may play central roles in the control of ripening in the grape berry [52]. As a support, Kühn et al. [53] investigated the aminocyclopropane-1-carboxylate (ACC) synthases genes in first developed grape berries, which pathway is related to ethylene signaling. For $C D P K$ genes, several genes including AtCPK16 [54], NtCDPK2 [55] and LeCDPK2 [56] were reported to phosphorylate ACC synthases to trigger ethylene biosynthesis and accumulation. Coincidence is that $V p C D P K 9$ and $V p C D P K 13$, as homologs of NtCDPK2 and LeCDPK2, are positively regulated under ethylene treatment, indicating their possible functions involved in ethylene signaling. However, $C D P K s$ functions in plants growth and development process still need more experimental evidence to deeply understand their biological roles and the pathways they involved in. 


\section{Conclusions}

So far, little systematic analysis of the CDPK family has been reported in grapevine, and the functions of most $C D P K$ genes remain unclear. However, accumulating evidence indicates that CDPKs play important roles in response to a broad variety of abiotic and biotic stresses and biological processes. In this paper, genome-wide identification, evolutionary, and expression analyses were carried out to provide a framework for further analysis of grape CDPK genes in defining their biological functions and pathways during stress responses as well as growth and development. Expression profiles showed grape $C D P K$ genes respond to various stresses and hormone treatments; moreover, analysis of CDPK subcellular localization gave evidence as to their functions. Comparisons of the grape and Arabidopsis genomes and expression profiles provide novel insights into the functions of less well-studied genes according to their betterunderstood homologs. By prediction and experimental data, we speculated that $C D P K$ gene family might participate in responses to pathogen, cold, heat and salt stress, and the related biological processes might covering the regulation of gene expression, control of the ion channel, regulation of the enzyme activity, mediation of the cross-talk between signaling pathways and so on. These observations may lay the foundation for future functional analysis of grape CDPK genes to unravel their biological roles.

\section{Methods}

\section{Identification of grape CDPK genes}

To identify the CDPK genes in grapevine, we downloaded the Hidden Markov Model (HMM) profiles of the core protein kinase domain (PF00069) and EF-hand domain (EF-hand_7, PF13499) from Pfam database (http://pfam.xfam.org/). Then we performed a BLAST-P search in the Grape Genome Database (12X) (http://www.genoscope.cns.fr/externe/GenomeBrowser/Vitis/) using the HMM profiles as queries with e-value of 0.01 . We also performed BLAST-P searches at NCBI using full-length amino acid sequences of the primarily identified grape $C D P K$ genes and chose the candidates of e-value lower than $1 \mathrm{e}^{-60}$. All putative $C D P K$ genes were manually verified with the InterProScan program (http://www.ebi.ac.uk/Tools/pfa/ iprscan/) to confirm their completeness and existence of the core domains. Among those with alternative splice variants, we selected the longest variant for further analysis. Sequences of Arabidopsis, rice, maize, and poplar $C D P K$ genes were obtained from the Arabidopsis Information Resource (TAIR, https://www.arabidopsis.org/), rice genome database in TIGR (http://rice.tigr.org), maize genome database (http://www.maizesequence.org/index.html), and Phytozome (http://www.phytozome.net/), respectively.

\section{Chromosomal localization and synteny analysis}

Grape CDPK genes were mapped to chromosomes by identifying their chromosomal locations, as obtained from the Grape Genome Database (12 X) (http://www.genoscope.cns.fr/externe/GenomeBrowser/Vitis/) and NCBI Map Viewer (http://www.ncbi.nlm.nih.gov/mapview/). The segmental and tandem duplication regions, as well as chromosomal location, were established using PLAZA v3.0 Dicots (http://bioinformatics.psb.ugent.be/plaza/versions/plaza_v3_dicots/). For synteny analysis, synteny blocks within the grape genome and between grape and Arabidopsis genomes were downloaded from the Plant Genome Duplication Database and visualized using Circos (http://circos.ca/).

\section{Gene structure and phylogenetic analysis}

Myristoylation and palmitoylation sites were predicted by Myristoylator (http://web.expasy.org/myristoylator/) and CSS-Palm 3.0 (http://csspalm.biocuckoo.org/), respectively. The intron-exon organization analysis was carried out using GSDS 2.0 (http://gsds.cbi.pku.edu.cn/) by alignment of the cDNA sequences with their corresponding genomic DNA sequences, and the results were consistent with the phylogenetic analysis. Multiple alignments of the identified grape CDPK amino acid sequences were performed using ClustalX. The phylogenetic tree was constructed with MEGA5.0 using the Neighbor-Joining method and the bootstrap test carried out with 1,000 replicates [57].

\section{Plant material and treatments}

The Chinese wild grapevine $V$. pseudoreticulata accession Baihe-35-1 was grown in the grapevine germplasm resources greenhouse of Northwest A\&F University in China, at temperatures of 22 to $27{ }^{\circ} \mathrm{C}$, relative humidity of 60 to $80 \%$, and without supplemental lighting. When shoots of vines were $40-50 \mathrm{~cm}$ in length, the third and fourth fully expanded young grapevine leaves beneath the apex were selected for treatments. Plants of Arabidopsis thaliana ecotype Col-0 were grown at $22{ }^{\circ} \mathrm{C}$, $75 \%$ relative humidity, and under short-day $(8 \mathrm{~h} \mathrm{light}$ at $125 \mu \mathrm{mol} \cdot \mathrm{m}^{-2} \cdot \mathrm{s}^{-1}, 16 \mathrm{~h}$ dark) conditions for 4 to 5 weeks before transformation.

A grapevine powdery mildew (Erysiphe necator) isolate NAFU1 (GenBank accession no. KJ539202) was collected from a vineyard in Northwest China, and maintained on the leaves of $V$. vinifera $\mathrm{cv}$. Thompson seedless, which was soil-grown in pots. The pathogen was sub-cultured onto fresh leaves every twenty days. The leaves of Baihe-35-1 were inoculated by touching the adaxial epidermis with sporulating colonies on the surface of the 'Thompson Seedless' infected leaves. Plants were then incubated in the greenhouse. Inoculated leaves were collected at $0,6,24,48,72,96,120$, 
and $144 \mathrm{~h}$ post-inoculation (hpi). Inoculations were repeated three times.

For salt stress treatments, four-month-old soil-grown plants were irrigated with $300 \mathrm{mM} \mathrm{NaCl}$. Treated leaves were collected at $0,0.5,2,4,8,12,24$, and 48 h posttreatment (hpt). For cold treatment, plants were first grown at $22{ }^{\circ} \mathrm{C}$, and then transferred to $4{ }^{\circ} \mathrm{C}$, and treated leaves were collected at $0,0.5,2,4,8,12,24$, and $48 \mathrm{~h}$ post-treatment (hpt). For high temperature treatment, plants were first grown at $22{ }^{\circ} \mathrm{C}$, and then transferred to $42{ }^{\circ} \mathrm{C}$, and treated leaves were collected at $0,0.5,2,4,8$, 12, 24, and $48 \mathrm{~h}$ post-treatment (hpt). The leaves of Baihe-35-1 were sprayed with solution of $0.1 \mathrm{mM}$ abscisic acid (ABA), $1 \mathrm{mM}$ salicylic acid (SA), $0.1 \mathrm{mM}$ methyl jasmonate (MeJA), or $0.5 \mathrm{~g} / \mathrm{L}$ ethephon (Eth), and then collected for RNA isolation. The treated leaves were collected at $0,2,4,8,12,24$, and $48 \mathrm{~h}$ post-treatment (hpt). Another set of control plants were similarly treated with distilled water. All plants were treated in the light and three independent experiments were performed.

\section{Reverse transcription quantitative PCR}

Total RNA of grape leaves was extracted using the E.Z.N.A. Plant RNA Kit (Omega, Guangzhou, China) according to the manufacturer's instructions. First-strand cDNA was synthesized from $2 \mu \mathrm{g}$ total RNA using PrimeScript RTase (Takara, Dalian, China). Quantitative PCR (qPCR) was carried out using SYBR green (Takara, Dalian, China) on an IQ5 real time PCR machine (Bio-Rad, Hercules, CA, USA) according to the manufacturer's instructions. Thermal cycling consisted of a hold at $95{ }^{\circ} \mathrm{C}$ for $30 \mathrm{~s}$, followed by 40 cycles of $95{ }^{\circ} \mathrm{C}$ for $30 \mathrm{~s}$ and $58^{\circ} \mathrm{C}$ for $30 \mathrm{~s}$. After amplification, samples were kept at $50{ }^{\circ} \mathrm{C}$ for $1 \mathrm{~min}$ and the temperature was raised gradually by $0.5{ }^{\circ} \mathrm{C}$ every $10 \mathrm{~s}$ to perform the melt-curve analysis. Grape VpActin (accession no. AY680701) was amplified as an internal control. All reactions were performed in triplicate in each experiment and three biological repeats were conducted. Primers used for RT-qPCR are listed in Additional file 11. Each relative expression level was analyzed with IQ5 software using the Normalized Expression method ( $2^{-\triangle \triangle C T}$ method). Expressional data consist of three replicated treatments and controls, which were calculated as 2 -log-based values and were divided by the control.

\section{Statistical analysis}

The statistical analysis was performed with the SPSS 19.0 software. The data were showed as mean value \pm $\mathrm{SD}$. We examined the homoscedasticity of our data by F-test. The significance of the differential expression between treatments and controls $(0 \mathrm{hpt})$ was verified by performing Student's $t$-test. $\mathrm{p}<0.05$ and $\mathrm{p}<0.01$ were taken as statistically significant or highly significant, respectively. The biological significance of RPKM was set as a fold change greater than 2-fold or less than 0.5fold.

\section{Subcellular localization of VpCDPK}

The predicted full-length coding sequences of grape $V p C D P K$ genes, including $V p C D P K 2, \quad V p C D P K 3$, $V p C D P K 5, V p C D P K 9, V p C D P K 10$, and $V p C D P K 11$, were amplified by high-fidelity Taq HS-mediated PCR from cDNA of the Chinese wild grapevine $V$. pseudoreticulata accession Baihe-35-1 leaves. The amplified PCR products were digested with SalI and XhoI and fused inframe with GFP in the SalI and XhoI site of the pBI221 vector containing the CaMV $35 S$ promoter (Clontech, Beijing, China) resulting in plasmids pVpCDPKs-GFP. Primers used for cloning genes and for constructing vectors are shown in Additional file 11.

For transient expression of VpCDPKs-GFP in Arabidopsis mesophyll protoplasts, DNA of the corresponding pVpCDPK-GFP plasmids was transformed into Col-0 leaves using a previously described method [58]. After transformation, Col-0 leaves were kept in darkness at room temperature for $16-18 \mathrm{~h}$ before examination by fluorescence microscopy. Images were acquired using an Olympus BX-51 inverted fluorescence microscope (Olympus, Japan). The image data were processed using Adobe Photoshop (Mountain View, CA, USA). All transient expression assays were repeated at least three times.

\section{Availability of supporting data}

Phylogenetic data (alignments and phylogenetic trees) supporting the results of this article have been deposited in TreeBASE respository and is available under the URL http://purl.org/phylo/treebase/phylows/study/

TB2:S17752.

Sequence data of the isolated $V p C D P K$ genes in this article can be found in GenBank (http://www.ncbi.nlm.nih.gov) under the accessions of KR153945- KR153946 and KR153948- KR153951.

\section{Additional files}

Additional file 1: The syntenic relationships among grape and Arabidopsis CDPK genes.

Additional file 2: The CDPKs amino acid sequences used to construct the phylogenetic tree.

Additional file 3: Detailed expression profiling of the remaining 15 $V p C D P K$ genes during powdery mildew infection. Detailed expression levels were measured by RT-qPCR. Grape Actin1 (GenBank Accession number AY680701) was used as an internal control. The experiments were repeated three times and gave consistent results. Mean values and SDs were obtained from three biological and three technical replicates. The data were showed as mean value \pm SD. * and ** 
represent statistically significant $(p<0.05)$ or highly significant $(p<0.01)$, respectively.

Additional file 4: Detailed expression profiling of the remaining 15 $V p C D P K$ genes under $\mathrm{NaCl}$ treatment. Detailed expression levels were measured by RT-qPCR. Grape Actin1 (GenBank Accession number AY680701) was used as an internal control. The experiments were repeated three times and gave consistent results. Mean values and SDs were obtained from three biological and three technical replicates. The data were showed as mean value $\pm \mathrm{SD}$. ${ }^{*}$ and ${ }^{* *}$ represent statistically significant $(p<0.05)$ or highly significant $(p<0.01)$, respectively.

Additional file 5: Detailed expression profiling of the remaining 15 $V p C D P K$ genes under $4^{\circ} \mathrm{C}$ treatment. Detailed expression levels were measured by RT-qPCR. Actin 1 (GenBank Accession number AY680701) was used as an internal control. The experiments were repeated three times and gave consistent results. Mean values and SDs were obtained from three biological and three technical replicates. The data were showed as mean value $\pm S D .{ }^{*}$ and ${ }^{* *}$ represent statistically significant $(p<0.05)$ or highly significant $(p<0.01)$, respectively.

\section{Additional file 6: Detailed expression profiling of the remaining 15} $V p C D P K$ genes under $42^{\circ} \mathrm{C}$ treatment. Detailed expression levels were measured by RT-qPCR. Actin1 (GenBank Accession number AY680701) was used as an internal control. The experiments were repeated three times and gave consistent results. Mean values and SDs were obtained from three biological and three technical replicates. The data were showed as mean value \pm SD. ${ }^{*}$ and ${ }^{* *}$ represent statistically significant $(p<0.05)$ or highly significant $(p<0.01)$, respectively.

\section{Additional file 7: Detailed expression profiling of the rest} remaining $V p C D P K$ genes under $A B A$ treatment. Detailed expression levels were measured by RT-qPCR. Actin 1 (GenBank Accession number AY680701) was used as an internal control. The experiments were repeated three times and gave consistent results. Mean values and SDs were obtained from three biological and three technical replicates. The data were showed as mean value $\pm S D$. ${ }^{*}$ and ${ }^{* *}$ represent statistically significant $(p<0.05)$ or highly significant $(p<0.01)$, respectively.

Additional file 8: Detailed expression profiling of the remaining 15 $V p C D P K$ genes under SA treatment. Detailed expression levels were measured by RT-qPCR. Actin1 (GenBank Accession number AY680701) was used as an internal control. The experiments were repeated three times and gave consistent results. Mean values and SDs were obtained from three biological and three technical replicates. The data were showed as mean value $\pm \mathrm{SD} .{ }^{*}$ and ${ }^{* *}$ represent statistically significant $(p<0.05)$ or highly significant $(p<0.01)$, respectively.

\section{Additional file 9: Detailed expression profiling of the remaining 15} $V p C D P K$ genes under MeJA treatment. Detailed expression levels were measured by RT-qPCR. Actin1 (GenBank Accession number AY680701) was used as an internal control. The experiments were repeated three times and gave consistent results. Mean values and SDs were obtained from three biological and three technical replicates. The data were showed as mean value \pm SD. ${ }^{*}$ and ${ }^{* *}$ represent statistically significant $(p<0.05)$ or highly significant $(p<0.01)$, respectively. (TIFF $4982 \mathrm{~kb})$

\section{Additional file 10: Detailed expression profiling of the remaining} 15 VpCDPK genes under ethylene treatment. Detailed expression levels were measured by RT-qPCR. Actin 1 (GenBank Accession number AY680701) was used as an internal control. The experiments were repeated three times and gave consistent results. Mean values and SDs were obtained from three biological and three technical replicates. The data were showed as mean value $\pm S D$. * and ** represent statistically significant $(p<0.05)$ or highly significant $(p<0.01)$, respectively.

\section{Additional file 11: Primers used in this study.}

\section{Abbreviations}

CDPK: Calcium-dependent protein kinase; RT-qPCR: Reverse transcriptionquantitative polymerase chain reaction; GFP: Green fluorescent protein; ABA: Abscisic acid; SA: Salicylic acid; MeJA: Methyl jasmonate; ETH: Ethylene; PM: Powdery mildew; Hpi: Hours post inoculation; Hpt: Hours post treatment.

\section{Competing interests}

The authors declare that they have no competing interests.

\section{Authors' contributions}

YQW, KZ and YTH conceived and designed the research. KZ and YZ carried out bioinformatics analyses. YTH performed all treatments and RT-qPCR tests, with assistance of YRG and YFM. FLZ, YH and KZ carried out subcellular localization experiments. KZ and YTH analysed and interpreted the data, and performed statistical analysis. KZ draft the manuscript and YQW revised it. YJW contributed with consultation. All authors read and approved the final manuscript.

\section{Acknowledgements}

The authors are grateful to Dr. Wen-ming Wang (Sichuan Agricultural University) for critical review of this manuscript and to Dr. Guang-lin Li (Shaanxi Normal University) for preparing the phylogenetic data profiles deposited to TreeBASE. We also thank Dr. Jennifer Mach for her useful comments and language editing. The authors would also like to thank the anonymous reviewers for comments on the manuscript. This work was supported by the National Natural Science Foundation of China (Grant No. 31372022, 31071772), and the program for Innovative Research Team of Grape Germplasm Resources and Breeding (2013KCT-25).

\section{Author details}

'State Key Laboratory of Crop Stress Biology for Arid Areas and College of Horticulture, Northwest A\&F University, Yangling 712100Shaanxi, People's Republic of China. ${ }^{2}$ Key Laboratory of Horticultural Plant Biology and Germplasm Innovation in Northwest China, Ministry of Agriculture, Yangling 712100Shaanxi, People's Republic of China. ${ }^{3}$ Boyce Thompson Institute for Plant Research, Cornell University, Ithaca, NY 14853, USA.

Received: 12 January 2015 Accepted: 15 June 2015 Published online: 30 June 2015

\section{References}

1. Sanders D, Brownlee C, Harper JF. Communicating with calcium. Plant Cell. 1999;11:691-706.

2. Kudla J, Batistic O, Hashimoto K. Calcium Signals: The Lead Currency of Plant Information Processing. Plant Cell. 2010;22:541-63.

3. Snedden WA, Fromm H. Calmodulin, calmodulin-related proteins and plant responses to the environment. Trends Plant Sci. 1998:3:299-304

4. Luan S, Kudla J, Rodriguez-Concepcion M, Yalovsky S, Gruissem W. Calmodulins and calcineurin B-like proteins: Calcium sensors for specific signal response coupling in plants. Plant Cell. 2002;14:S389-400.

5. Sanders D, Pelloux J, Brownlee C, Harper JF. Calcium at the crossroads of signaling. Plant Cell. 2002;14:S401-17.

6. Harmon AC, Gribskov M, Gubrium E, Harper JF. The CDPK superfamily of protein kinases. New Phytol. 2001;151:175-83.

7. Ludwig AA, Romeis T, Jones JDG. CDPK-mediated signalling pathways: specificity and cross-talk. J Exp Bot. 2004;55:181-8.

8. Boudsocq M, Willmann MR, McCormack M, Lee H, Shan LB, He P, et al. Differential innate immune signalling via $\mathrm{Ca}^{2+}$ sensor protein kinases. Nature. 2010;464:418-U116.

9. Cheng SH, Willmann MR, Chen HC, Sheen J. Calcium signaling through protein kinases. The Arabidopsis calcium-dependent protein kinase gene family. Plant Physiol. 2002;129:469-85.

10. Ray S, Agarwal P, Arora R, Kapoor S, Tyagi AK. Expression analysis of calcium-dependent protein kinase gene family during reproductive development and abiotic stress conditions in rice (Oryza sativa L. ssp indica) Mol Genet Genomics. 2007;278:493-505.

11. Li AL, Zhu YF, Tan XM, Wang X, Wei B, Guo HZ, et al. Evolutionary and functional study of the CDPK gene family in wheat (Triticum aestivum L.) Plant Mol Biol. 2008;66:429-43.

12. Kong XP, Lv W, Jiang SS, Zhang D, Cai GH, Pan JW, et al. Genome-wide identification and expression analysis of calcium-dependent protein kinase in maize. BMC Genomics. 2013;14:433.

13. Zuo R, Hu RB, Chai GH, Xu ML, Qi G, Kong YZ, et al. Genome-wide identification, classification, and expression analysis of CDPK and its closely related gene families in poplar (Populus trichocarpa). Mol Biol Rep. 2013;40:2645-62.

14. Davletova S, Meszaros T, Miskolczi P, Oberschall A, Torok K, Magyar Z, et al. Auxin and heat shock activation of a novel member of the calmodulin like domain protein kinase gene family in cultured alfalfa cells. J Exp Bot. 2001;52:215-21. 
15. Raices M, Ulloa RM, Maclntosh GC, Crespi M, Tellez-Inon MT. StCDPK1 is expressed in potato stolon tips and is induced by high sucrose concentration. J Exp Bot. 2003;54:2589-91.

16. Llop-Tous I, Dominguez-Puigjaner E, Vendrell M. Characterization of a strawberry cDNA clone homologous to calcium-dependent protein kinases that is expressed during fruit ripening and affected by low temperature. J Exp Bot. 2002;53:2283-5.

17. Chico JM, Raices M, Tellez-Inon MT, Ulloa RM. A calcium-dependent protein kinase is systemically induced upon wounding in tomato plants. Plant Physiol. 2002;128:256-70.

18. Xing T, Wang XJ, Malik K, Miki BL. Ectopic expression of an Arabidopsis calmodulin-like domain protein kinase-enhanced NADPH oxidase activity and oxidative burst in tomato protoplasts. Mol Plant Microbe In 2001;14:1261-4.

19. Coca M, Segundo B. AtCPK1 calcium-dependent protein kinase mediates pathogen resistance in Arabidopsis. Plant J. 2010;63:526-40.

20. Cheng SH, Sheen J, Gerrish C, Bolwell GP. Molecular identification of phenylalanine ammonia-lyase as a substrate of a specific constitutively active Arabidopsis CDPK expressed in maize protoplasts. Febs Lett. 2001;503:185-8

21. Dubiella U, Seybold H, Durian G, Komander E, Lassig R, Witte $\mathrm{CP}$, et al. Calcium-dependent protein kinase/NADPH oxidase activation circuit is required for rapid defense signal propagation. Proc Natl Acad Sci U S A 2013;110:8744-9.

22. Zhu SY, Yu XC, Wang XJ, Zhao R, Li Y, Fan RC, et al. Two calcium-dependent protein kinases, CPK4 and CPK11, regulate abscisic acid signal transduction in Arabidopsis. Plant Cell. 2007;19:3019-36.

23. Mori IC, Murata Y, Yang Y, Munemasa S, Wang YF, Andreoli S. CPK6 and CPK3 Function in ABA Regulation of Guard Cell S-Type Anion- and $\mathrm{Ca}^{2+}$ Permeable Channels and Stomatal Closure. PLoS Biol. 2006;4, e327.

24. Ma SY, Wu WH. AtCPK23 functions in Arabidopsis responses to drought and salt stresses. Plant Mol Biol. 2007:65:511-8.

25. Choi HI, Park HJ, Park JH, Kim S, Im MY, Seo HH, et al. Arabidopsis calciumdependent protein kinase AtCPK32 interacts with ABF4, a transcriptional regulator of abscisic acid-responsive gene expression, and modulates its activity. Plant Physiol. 2005;139:1750-61

26. Zhao R, Sun HL, Mei C, Wang XJ, Yan L, Liu R, et al. The Arabidopsis Ca ${ }^{2}$ ${ }^{+}$-dependent protein kinase CPK12 negatively regulates abscisic acid signaling in seed germination and post-germination growth. New Phytol. 2011;192:61-73.

27. Munemasa S, Hossain MA, Nakamura Y, Mori IC, Murata Y. The Arabidopsis Calcium-Dependent Protein Kinase, CPK6. Functions as a Positive Regulato of Methyl Jasmonate Signaling in Guard Cells. Plant Physiol. 2011;155:553-61.

28. Luo XJ, Chen ZZ, Gao JP, Gong ZZ. Abscisic acid inhibits root growth in Arabidopsis through ethylene biosynthesis. Plant J. 2014;79:44-55.

29. Wang YJ, Liu Y, He P, Chen J, Lamicanra O, Lu J. Evaluation of foliar resistance to Uncinula necator in Chinese wild Vitis species. Vitis. 1995:34:159-64.

30. Wen YQ, Wang XP, Xiao SY, Wang YJ. Ectopic expression of VpALDH2B4, a novel aldehyde dehydrogenase gene from Chinese wild grapevine (Vitis pseudoreticulata), enhances resistance to mildew pathogens and salt stress in Arabidopsis. Planta. 2012;236:525-39.

31. Chen F, Fasoli M, Tornielli GB, Dal Santo S, Pezzotti M, Zhang LS, et al. The Evolutionary History and Diverse Physiological Roles of the Grapevine Calcium-Dependent Protein Kinase Gene Family. PLoS One. 2013:8:e80818.

32. Dubrovina AS, Kiselev KV, Khristenko VS. Expression of calcium-dependent protein kinase (CDPK) genes under abiotic stress conditions in wild-growing grapevine Vitis amurensis. J Plant Physiol. 2013;170:1491-500.

33. Boudsocq M, Sheen J. CDPKs in immune and stress signaling. Trends Plant Sci. 2013;18:30-40

34. Zhang JQ, Guo C, Liu GF, Li ZN, Li XM, Bao MZ. Genetic alteration with variable intron/exon organization amongst five PI-homoeologous genes in Platanus acerifolia. Gene. 2011;473:82-91.

35. Vision TJ, Brown DG, Tanksley SD. The origins of genomic duplications in Arabidopsis. Science. 2000;290:2114-7.

36. Hughes AL. The Evolution of Functionally Novel Proteins after Gene Duplication. P Roy Soc B-Biol Sci. 1994;256:119-24.

37. Jaillon $\mathrm{O}$ et al. The grapevine genome sequence suggests ancestral hexaploidization in major angiosperm phyla. Nature. 2007;449:463-7.
38. Dammann C, Ichida A, Hong BM, Romanowsky SM, Hrabak EM, Harmon AC, et al. Subcellular targeting of nine calcium-dependent protein kinase isoforms from Arabidopsis. Plant Physiol. 2003;132:1840-8.

39. Latz A, Mehlmer N, Zapf S, Mueller TD, Wurzinger B, Pfister B, et al. Salt Stress Triggers Phosphorylation of the Arabidopsis Vacuolar K Channel TPK by Calcium-Dependent Protein Kinases (CDPKs). Mol Plant. 2013;6:1274-89.

40. Fujita M, Fujita Y, Noutoshi Y, Takahashi F, Narusaka Y, Yamaguchi-Shinozaki $K$, et al. Crosstalk between abiotic and biotic stress responses: a current view from the points of convergence in the stress signaling networks. Curr Opin Plant Biol. 2006;9:436-42.

41. Zou JJ, Wei FJ, Wang C, Wu JJ, Ratnasekera D, Liu WX, et al. Arabidopsis Calcium-Dependent Protein Kinase CPK10 Functions in Abscisic Acid- and $\mathrm{Ca}^{2+}$-Mediated Stomatal Regulation in Response to Drought Stress. Plant Physiol. 2010;154:1232-43.

42. Xu GX, Guo CC, Shan HY, Kong HZ. Divergence of duplicate genes in exon-intron structure. Proc Natl Acad Sci U S A. 2012;109:1187-92.

43. Hamel LP, Sheen J, Seguin A. Ancient signals: comparative genomics of green plant CDPKs. Trends Plant Sci. 2014;19:79-89.

44. Cannon SB, Mitra A, Baumgarten A, Young ND, May G. The roles of segmental and tandem gene duplication in the evolution of large gene families in Arabidopsis thaliana. BMC Plant Biol. 2004:4:10.

45. Ghiurcuta CG, Moret BME. Evaluating synteny for improved comparative studies. Bioinformatics. 2014;30:9-18.

46. Bohmer M, Romeis T. A chemical-genetic approach to elucidate protein kinase function in planta. Plant Mol Biol. 2007;65:817-27.

47. Lu SX, Hrabak EM. The myristoylated amino-terminus of an Arabidopsis calcium-dependent protein kinase mediates plasma membrane localization. Plant Mol Biol. 2013:82:267-78.

48. Gao X, He P. Nuclear dynamics of Arabidopsis calcium-dependent protein kinases in effector- triggered immunity. Plant Signal Behav. 2013;8, e23868.

49. Ma Y, Zhao YC, Walker RK, Berkowitz GA. Molecular Steps in the Immune Signaling Pathway Evoked by Plant Elicitor Peptides: $\mathrm{Ca}^{2+}$-Dependent Protein Kinases, Nitric Oxide, and Reactive Oxygen Species Are Downstream from the Early $\mathrm{Ca}^{2+}$ Signal. Plant Physiol. 2013:163:1459-71.

50. Ronzier E, Corratge-Faillie C, Sanchez F, Prado K, Briere C, Leonhardt N, et al. CPK13, a noncanonical $\mathrm{Ca}^{2+}$-dependent protein kinase, specifically inhibits KAT2 and KAT1 shaker $\mathrm{K}^{+}$channels and reduces stomatal opening. Plant Physiol. 2014;166:314-26.

51. Gao X, Chen X, Lin W, Chen S, Lu D. Bifurcation of Arabidopsis NLR Immune Signaling via $\mathrm{Ca}^{2+}$-Dependent Protein Kinases. PLoS Pathol. 2013;9 e1003127.

52. Kühn N, Guan L, Dai ZW, Wu BH, Lauvergeat V, Gomès E, et al. Berry ripening: recently heard through the grapevine. J Exp Bot. 2014;65:4543-59.

53. Kühn N, Abello C, Godoy F, Delrot S, Patricio AJ. Differential Behavior within a Grapevine Cluster: Decreased Ethylene-Related Gene Expression Dependent on Auxin Transport Is Correlated with Low Abscission of First Developed Berries. PLoS One. 2014:9, e111258.

54. Huang S J, Chang C L, Wang P H, Tsai MC, Hsu PH, Chang F. A type III ACC synthase, ACS7, is involved in root gravitropism in Arabidopsis thaliana. J Exp Bot. 2013: ert241.

55. Ludwig AA, Saitoh H, Felix G, Freymark G, Miersch O, Wasternack C, et al. Ethylene-mediated cross-talk between calcium-dependent protein kinase and MAPK signaling controls stress responses in plants. Proc Natl Acad Sci U S A. 2005;102:10736-41.

56. Kamiyoshihara Y, Iwata M, Fukaya T, Tatsuki M, Mori H. Turnover of LeACS2, a wound-inducible 1-aminocyclopropane-1-carboxylic acid synthase in tomato, is regulated by phosphorylation/ dephosphorylation. The Plant J. 2010;64:140-50

57. Tamura K, Peterson D, Peterson N, Stecher G, Nei M, Kumar S. MEGA5: Molecular Evolutionary Genetics Analysis using Maximum Likelihood, Evolutionary Distance, and Maximum Parsimony Methods. Mol Biol Evol. 2011;28:2731-9.

58. Yoo SD, Cho YH, Sheen J. Arabidopsis mesophyll protoplasts: a versatile cell system for transient gene expression analysis. Nat Protocols. 2007;2:1565-72. 